\title{
Vector-valued risk measure processes
}

\author{
Imen Ben Tahar \\ CEREMADE, CNRS UMR 7534 \\ Paris Dauphine University, France \\ imen@ceremade.dauphine.fr
}

\author{
Emmanuel Lépinette \\ CEREMADE, CNRS UMR 7534 \\ Paris Dauphine University, France \\ emmanuel.lepinette@ceremade.dauphine.fr
}

\begin{abstract}
Introduced by Artzner, Delbaen, Eber and Heath (1998) the axiomatic characterization of a static coherent risk measure was extended by Jouini, Meddeb and Touzi (2004) in a multi-dimensional setting to the concept of vector-valued risk measures. In this paper, we propose a dynamic version of the vector-valued risk measures in a continuous-time framework. Particular attention is devoted to the choice of a convenient risk space. We provide dual characterization results and examples of vector valued risk measure processes.
\end{abstract}

Key words vector-valued risk measure, coherent risk measure, dynamic risk measure, dual representation, transaction costs, partial order

Mathematics Subject Classification (2000) 60G44

JEL Classification G11 · G13

\section{Introduction}

Artzner, Delbaen Eber and Heath initiated in their seminal paper [1] the concept of Monetary coherent risk measures. In their approach, a financial position is identified with a real-valued random variable $X \in L^{\infty}(\Omega, \mathcal{F}, P)$ which models the profit and loss of the position at some final date $T$. The risk measure $\rho(X)$ of the position $X$ is defined as the "extra cash" requirement that has to be invested at the beginning of the period in some secure instrument so that the resulting position is acceptable with regard to some specified criterion, that is: $X+\rho(X) \in \mathcal{A}$ where $\mathcal{A}$ is a given subset of $L^{\infty}(\Omega, \mathcal{F}, P)$ called the acceptance set. A set of axioms, namely: (i) subadditivity, (ii) monotonicity, (iii) positive homogeneity and (iv) translation invariance, are imposed on the risk measure $\rho($.$) - or equivalently on the acceptance set \mathcal{A}$ - to guarantee economic coherence. This pioneering approach [1], [4] has been extended by Jouini, Meddeb and Touzi [9] to the context of frictious financial markets where financial positions cannot 
be aggregated through a 1-dimensional random variable, but are modeled through $\mathbf{R}^{d}$ valued random vectors. This is indeed the case if we consider realistic situations where investors have access to different markets and form multi-asset portfolios in the presence of frictions such as transaction costs, liquidity problems, irreversible transfers, etc. Let us describe briefly the model of [9] on which subsequent papers on vector-valued risk measure [6], [7], as well as our present exposition, are based: the authors consider essentially bounded $\mathbf{R}^{d}$-valued random variables $X \in L_{d}^{\infty}(\Omega, \mathcal{F}, P)$ and assume that the risk space $L_{d}^{\infty}(\Omega, \mathcal{F}, P)$ is endowed by a partial order relation $\preceq$ accounting for the frictions in the market. A vector-valued risk measure is defined as a set-valued map $\rho$ which associates with each risk $X$ a subset $\rho(X) \subseteq \mathbf{R}^{n}$; an element $x \in \rho(X)$ is interpreted as a deterministic portfolio, formed using $n$ reference instruments, which can be invested at the beginning of the period to control the risk of $X$. The above mentioned coherency axioms (i)-(iv) are adapted to this set-valued context and the authors provide dual representation results for coherent vector-valued risk measures which are consistent with the representation theorems for coherent real-valued risk-measures.

In this paper, we are mainly concerned with the extension of the concept of vectorvalued risk measures to a dynamic setting. As days go long, it seems natural to update the risk measure of a given position taking into account new information. Dynamic risk measures have already been introduced and studied in the 1-dimensional setting in the previous literature, see for example [12], [2], [8]. In higher dimension, very few results exist, to our knowledge, the only attempt to define dynamic set-valued risk measures is due to [5] and is based on the classical $L^{p}$-spaces approach and the geometrical formalism of [13].

One of the main contributions of our paper is to define the dynamic risk measure on a risk space different from the classical $L^{p}$ spaces and which, to our opinion, better suits financial models with transactions costs, as the Kabanov and the Campi-Schachermayer models [10]. Our choice for the risk-space naturally arises from the partial order relation given by the solvency cones. This choice is based on a boundedness-concept adapted to the partial order relation. It does not depend on the choice of the probability measure (as the $L_{d}^{\infty}$-space), but allows to consider positions which are not essentially bounded, and this is a more realistic feature for applications. We leave for future research a more systematic analysis of the different consistence in time approaches to our model.

This paper is organized as follows. Section 2 is dedicated to the risk-space. In Section 3 we introduce the main concepts related to dynamic vector-valued risk process. Then, Section 4 is devoted to the dual characterization of vector-valued risk measure processes. Finally, examples are given in Section 5.

Notations: We shall denote by $x \cdot y$ the scalar product of $x, y \in \mathbf{R}^{n}$. For a vector $x \in \mathbf{R}^{n}, x^{i}$ denotes its $i^{\text {th }}$ component. We denote by $\mathbf{1}^{i}$ the vector of $\mathbf{R}^{n}$ defined by: $\mathbf{1}^{i i}=1$ and $\mathbf{1}^{i j}=0$ if $j \neq i$, and we set $\mathbf{1}:=\sum_{i=1}^{n} \mathbf{1}^{i}$. We set $\mathbf{R}_{++}:=(0, \infty)$.

Given $d \geq n$, we denote by $\mathbf{1}_{d, n}$ the vector of $\mathbf{R}^{d}$ whose all components are equal to zero except the $n$ first ones which are equal to 1 . If $x \in \mathbf{R}^{n}, \bar{x}$ denotes the vector of $\mathbf{R}^{d}$ 
defined by $\bar{x}:=\left(x, 0_{d-n}\right)$ where $0_{d-n}=(0, \cdots, 0) \in \mathbf{R}^{d-n}$. We denote by $\mathbf{R}^{n} \times 0_{d-n}$ the set $\left\{\bar{x}, x \in \mathbf{R}^{n}\right\}$.

For a set $A \subset \mathbf{R}^{n}$ we shall denote by $\mathbf{1}_{A}$ its indicator function, and by $\operatorname{cl}[A]$ its closure. If $A$ is a cone, $A^{\star}$ denotes its positive dual cone defined by

$$
A^{\star}:=\left\{y \in \mathbf{R}^{n}: x \cdot y \geq 0, x \in A\right\} .
$$

Finally we shall denote by $\mathcal{P}\left(\mathbf{R}^{n}\right)$ the collection of all subsets of $\mathbf{R}^{n}$.

\section{The setting}

\subsection{Basic definitions}

Throughout this paper, we fix a time horizon $T>0$ and we consider a stochastic basis $\left(\Omega, \mathcal{F}, \mathbb{F}=\left(\mathcal{F}_{t}\right)_{t \in[0, T]}, P\right)$ satisfying the usual conditions.

Following [10], we say that a map $\Gamma: \Omega \rightarrow \mathcal{P}\left(\mathbf{R}^{d}\right)$ is an $\mathcal{F}_{t}$-measurable random set if its graph $\left\{(\omega, x) \in \Omega \times \mathbf{R}^{n}: x \in \Gamma(\omega)\right\}$ is $\mathcal{F}_{t} \otimes \mathcal{B}\left(\mathbf{R}^{d}\right)$-measurable. The random set $\Gamma$ is said to be a closed (resp. closed convex) random set if for $P-a . e \omega, \Gamma(\omega)$ is a nonempty closed (resp. closed convex) subset of $\mathbf{R}^{d}$. An adapted random set process is a family $\left(\Gamma_{t}\right)_{t \in[0, T]}$ where for each $t \in[0, T], \Gamma_{t}: \Omega \rightarrow \mathcal{P}\left(\mathbf{R}^{d}\right)$ is an $\mathcal{F}_{t}$-measurable random set. We shall denote by $\mathcal{S}_{[0, T]}^{d}$ the set of adapted random set processes.

As usual, $L_{d}^{0}\left(\mathcal{F}_{t}\right)$ stands for the space of all measurable $\mathbf{R}^{d}$-valued random vectors and $L_{d}^{\infty}\left(\mathcal{F}_{t}\right)$ stands for the space of all measurable $\mathbf{R}^{d}$-valued random vectors with finite $L^{p}$-norm, $1 \leq p \leq \infty$. If $\Gamma: \Omega \rightarrow \mathcal{P}\left(\mathbf{R}^{d}\right)$ is an $\mathcal{F}_{t}$-random set, we shall denote by $L_{d}^{p}\left(\Gamma, \mathcal{F}_{t}\right)$ the set of random vectors $X \in L_{d}^{p}\left(\mathcal{F}_{t}\right)$ such that $X(\omega) \in \Gamma(\omega)$ for $P$-a.e. $\omega$.

In our setting, a random vector $X:\left(\Omega, \mathcal{F}_{T}, P\right) \rightarrow \mathbf{R}^{d}$ models the terminal value of some multi-asset financial portfolio.

Let us consider a set-valued $\mathcal{F}_{T}$-measurable random set $G_{T}$ satisfying the following Assumption G:

g1: for a.e. $\omega, G_{T}(\omega)$ is a closed convex cone of $\mathbf{R}^{d}$.

g2: $\mathbf{R}_{+}^{d} \subseteq G_{T}$ and $G_{T} \neq \mathbf{R}^{d}$ a.s.

g3: for a.e. $\omega, G_{T}(\omega)$ is a proper cone.

The random set $G_{T}$ induces on the space $L_{d}^{0}\left(\mathcal{F}_{T}\right)$ of terminal values a natural partial order relation $\geq_{G_{T}}$ by:

$$
X \geq_{G_{T}} Y \text { if and only if } X(\omega)-Y(\omega) \in G_{T}(\omega) \text { for a.e } \omega
$$

Example 2.1 Consider the model of financial market with proportional transaction costs described in [10]. The market is formed by $d$ assets $S^{1}, \cdots, S^{d}$ and trading is liable for proportional transaction costs: transferring, at time $t$, an amount $m \geq 0$ from asset $S^{i}$ to asset $S^{j}$ requires a transaction cost of $\lambda_{t}^{i j} m$. An agent's position at time $t$ can be described by a random vector $X_{t} \in R^{d}$ such that the $i^{t h}$ component $X_{t}^{i}$ represents the value of his position in asset $S^{i}$. The agent position, $X_{t}$, is said to be solvable iff 
it is possible to realize a transaction, i.e. transfers of certain amounts described by a $d \times d$ matrix $a=\left(a_{i j}\right)_{1 \leq i, j \leq d} \in \mathbf{R}_{+}^{d \times d}$ such that the resulting position has non-negative

components: $X_{t}^{i}+\sum_{j=1}^{d}\left(a_{j i}-\left(1+\lambda_{t}^{i j}\right) a_{i j}\right) \geq 0$. Hence, $X_{t}$ is solvable if and only if it is almost surely contained in the closed convex cone $K_{t}$ defined by

$$
K_{t}:=\left\{x \in \mathbf{R}^{d}: \exists\left(a_{i j}\right)_{1 \leq i, j \leq d} \in \mathbf{R}_{+}^{d \times d}, \forall i, x^{i}+\sum_{j=1}^{d}\left(a_{j i}-\left(1+\lambda_{t}^{i j}\right) a_{i j}\right) \geq 0\right\}
$$

We may consider the random cone $G_{T}:=K_{T}$. Notice that $G_{T}$ satisfies the Assumption G. In this context, if two financial positions $X_{T}$ and $Y_{T}$ are such that, $X_{T} \geq_{G_{T}} Y_{T}$, then clearly, $X_{T}$ is considered less risky than $Y_{T}$.

\subsection{The set of $G_{T}$-bounded positions}

In this paragraph we consider final positions $X_{T} \in L_{d}^{0}\left(\mathcal{F}_{T}\right)$ which are lower or upper bounded with respect to the preorder relation $\geq_{G_{T}}$. We consider the following subspaces of $L_{d}^{0}\left(\mathcal{F}_{T}\right)$.

$$
\begin{aligned}
L B_{G_{T}, n} & :=\left\{X: X \geq_{G_{T}}-c \mathbf{1}_{d, n} \text { for some } c \geq 0\right\}, \\
U B_{G_{T}, n} & :=\left\{X: c \mathbf{1}_{d, n} \geq_{G_{T}} X \text { for some } c \geq 0\right\},
\end{aligned}
$$

and the set of $G_{T}$-bounded positions:

$$
B_{G_{T}, n}:=L B_{G_{T}, n} \cap U B_{G_{T}, n} .
$$

If $\Gamma: \Omega \rightarrow \mathcal{P}\left(\mathbf{R}^{d}\right)$ is an $\mathcal{F}_{T^{-}}$-measurable random set, we shall denote denote $B_{G_{T}, n}\left(\Gamma, \mathcal{F}_{t}\right)$ the set of random vectors $\gamma \in B_{G_{T}, n} \cap L_{d}^{0}\left(\mathcal{F}_{t}\right)$ such that $\gamma \in \Gamma$ a.s.

Now, define the mapping $\|\cdot\|_{G_{T}, n}: B_{G_{T}, n} \rightarrow \mathbf{R}_{+}$by

$$
\|X\|_{G_{T}, n}:=\inf \left\{c \geq 0: c \mathbf{1}_{d, n} \geq_{G_{T}} X \geq_{G_{T}}-c \mathbf{1}_{d, n}\right\} .
$$

Since $G_{T}$ is cone-valued and contains $\mathbf{R}_{+}^{d}$, it is easy to verify that for each $X \in B_{G_{T}, n}$ :

$$
\left\{c \geq 0: c \mathbf{1}_{d, n} \geq_{G_{T}} X \geq_{G_{T}}-c \mathbf{1}_{d, n}\right\}=\left[\|X\|_{G_{T}, n},+\infty\right) .
$$

If $G_{T}$ is proper, i.e. if it satisfies the additional requirement: $G_{T} \cap\left(-G_{T}\right)=\{0\}$, then the mapping $\|\cdot\|_{G_{T}, n}$ defines a norm on $B_{G_{T}, n}$.

It is easy to verify the following property for the normed space $\left(B_{G_{T}, n},\|\cdot\|_{G_{T}, n}\right)$.

Proposition 2.2 Let $\left(X_{m}\right)_{m \geq 1}$ be a sequence of random vectors which converges in $\left(B_{G_{T}, n},\|\cdot\|_{G_{T}, n}\right)$ to $X$. Then the convergence holds almost surely.

Now, we are going to state the important Cauchy property for $\left(B_{G_{T}, n},\|\cdot\|_{G_{T}, n}\right)$.

Proposition 2.3 Assume that the cone $G_{T}$ is proper. Then, the vector space $B_{G_{T}, n}$ endowed with $\|\cdot\|_{G_{T}, n}$ is a Banach space. 
Proof. Consider a Cauchy sequence $\left(X_{m}\right)_{m \geq 1}$ in $\left(B_{G_{T}, n},\|\cdot\|_{G_{T}, n}\right)$. Define the sequence $k_{m}:=\left\|X_{m}\right\|_{G_{T}, n}$. Clearly $\left(k_{m}\right)_{m}$ is a Cauchy sequence in $\mathbf{R}$, hence it converges to some $k_{\infty} \in \mathbf{R}_{+}$.

1. First, we show that: $\xi:=\liminf \left|X_{m}\right|<\infty$ almost surely. We proceed by contradiction and assume that the set $\tilde{\Omega}:=\{\omega, \xi(\omega)=\infty\}$ has a positive measure. We define the sequence $\tilde{X}_{m}:=X_{m} /\left(\left|X_{m}\right| \vee 1\right)$. This sequence satisfies $\tilde{\xi}:=\lim \inf \left|\tilde{X}_{m}\right|<$ $\infty$ almost surely, then, by virtue of Lemma 2.1.2 [10], there exists an increasing sequence of integer-valued random variables $\left(\sigma_{m}\right)_{m \geq 1}$ such that $\tilde{X}_{\sigma_{m}}$ converges a.s. to some $\tilde{X} \in L_{d}^{0}\left(\mathcal{F}_{T}\right)$ with $|\tilde{X}|=1$ on $\tilde{\Omega}$. Notice that

$$
-\frac{k_{\sigma_{m}}}{\left|X_{\sigma_{m}}\right| \vee 1} \mathbf{1}_{d, n} \leq_{G_{T}} \tilde{X}_{\sigma_{m}} \leq_{G_{T}} \frac{k_{\sigma_{m}}}{\left|X_{\sigma_{m}}\right| \vee 1} \mathbf{1}_{d, n} .
$$

Letting $m \rightarrow \infty$, using the fact that $\left(k_{\sigma_{m}}\right)_{m}$ converges to $k_{\infty}$ almost surely and the fact that the cone $G_{T}$ is closed and proper we get that $\tilde{X}=0$ on $\tilde{\Omega}$ which is in contradiction with $|\tilde{X}|=1$ on the non-null set $\tilde{\Omega}$.

2. Since $\xi:=\liminf \left|X_{m}\right|<\infty$ almost surely, Lemma 2.1.2 [10] implies that there exists an increasing sequence of integer-valued random variables $\left(\alpha_{m}\right)_{m \geq 1}$ such that $X_{\alpha_{m}}$ converges a.s. to some $X_{\infty} \in L_{d}^{0}\left(\mathcal{F}_{T}\right)$. Moreover, the sequence $\alpha_{q}$ can be chosen so that $\alpha_{q} \geq q$. Now, letting $m \rightarrow \infty$ in the inequalities: $-k_{\alpha_{m}} \mathbf{1}_{d, n} \leq_{G_{T}} X_{\alpha_{m}} \leq_{G_{T}} k_{\alpha_{m}} \mathbf{1}_{d, n}$, we get: $-k_{\infty} \mathbf{1}_{d, n} \leq_{G_{T}} X_{\infty} \leq k_{\infty} \mathbf{1}_{d, n}$ and $X_{\infty} \in B_{G_{T}, n}$.

3. It remains to show that: $\lim _{m \rightarrow \infty}\left\|X_{m}-X_{\infty}\right\|_{G_{T}, n}=0$. For this, consider an arbitrary $\varepsilon>0$ and let $m_{\varepsilon} \geq 1$ such that: $\left\|X_{m}-X_{q}\right\|_{G_{T}, n} \leq \varepsilon$ for each $m, q \geq m_{\varepsilon}$. For each $q, m \geq m_{\varepsilon}$ we have $\alpha_{q} \geq q \geq m_{\varepsilon}$, and $X_{\infty}-X_{m}=X_{\infty}-X_{\alpha_{q}}+X_{\alpha_{q}}-X_{m}$ where: $-\varepsilon \mathbf{1}_{d, n} \leq_{G_{T}} X_{\alpha_{q}}-X_{m} \leq_{G_{T}} \varepsilon \mathbf{1}_{d, n}$, hence

$$
-\varepsilon \mathbf{1}_{d, n}+\left(X_{\infty}-X_{\alpha_{q}}\right) \leq_{G_{T}} \quad X_{\infty}-X_{m} \quad \leq_{G_{T}} \varepsilon \mathbf{1}_{d, n}+\left(X_{\infty}-X_{\alpha_{q}}\right)
$$

when $q \rightarrow \infty$, we get: $-\varepsilon \mathbf{1}_{d, n} \leq_{G_{T}} X_{\infty}-X_{m} \leq_{G_{T}} \varepsilon \mathbf{1}_{d, n}$ for each $m \geq m_{\varepsilon}$.

Remark 2.4 Clearly the set $L_{d}^{\infty}\left(\mathcal{F}_{T}\right) \subset B_{G_{T}, n}$. In general, the reverse inclusion does not hold. It is particularly the case in models where transactions costs are not uniformly bounded from below. As example, let us consider the financial market of Example 2.1 in the case where $d=2, \Omega=(0,+\infty)$ and the random transaction costs are such that $\lambda^{1,2}=\lambda^{2,1}:=\lambda: \omega \mapsto \omega$. Then an easy computation shows that the random variable $X$ given by

$$
X=\left(\begin{array}{c}
\frac{(2+\lambda)(1+\lambda)}{\left(\lambda^{2}+2 \lambda\right)} \\
-\frac{2+\lambda}{\left(\lambda^{2}+2 \lambda\right)}
\end{array}\right)
$$

satisfies $0 \leq_{G_{T}} X \leq_{G_{T}} \mathbf{1}_{2,2}$, hence $\|X\|_{G_{T}, 2} \leq 1$; on the other hand $X \notin L_{d}^{\infty}\left(\mathcal{F}_{T}\right)$, indeed $|X(\omega)|$ converges to $\infty$ as $\omega$ goes to 0 . Notice that in this example, if the transaction costs were bounded from below by a positive constant: $\inf _{\omega} \lambda(\omega)>0$, then we would have $L_{d}^{\infty}\left(\mathcal{F}_{T}\right)=B_{G_{T}, n}$. 


\section{Vector-valued Risk measure process}

\subsection{Definition and first properties}

Definition 3.1 Let $t \in[0, T]$. A subset $\mathcal{C} \subseteq B_{G_{T}, n}$ is called an $\mathcal{F}_{t}$-cone if for each $X \in \mathcal{C}$ and $\lambda \in L_{1}^{0}\left(\mathcal{F}_{t}\right)$ such that $\lambda \geq 0$ a.s. and $\lambda X \in B_{G_{T}, n}$, we have that $\lambda X \in \mathcal{C}$.

Definition $3.2(d, n)$-coherent risk process

$A(d, n)$-coherent risk process is a mapping $\rho$ defined on a convex $\mathcal{F}_{T}$-cone $D$ of $B_{G_{T}, n}$ with values on $\mathcal{S}_{[0, T]}^{n}$ satisfying the following statements.

A0) If $X \in D, \rho_{t}(X)$ is a closed $\mathcal{F}_{t}$-measurable random-set, and $0 \in \rho_{t}(0) \subsetneq \mathbf{R}^{n}$.

A1) If $X, Y \in D$ are such that $X \geq_{G_{T}} Y$, then $\rho_{t}(Y) \subseteq \rho_{t}(X)$ for each $t \in[0, T]$.

A2) If $X, Y \in D$, then $\rho_{t}(X)+\rho_{t}(Y) \subseteq \rho_{t}(X+Y)$ for each $t \in[0, T]$.

A3) If $X \in D$ and $\lambda \in L_{1}^{0}\left(\mathcal{F}_{t}\right)$ with $\lambda>0$ a.s. and $\lambda X \in D$, then $\rho_{t}(\lambda X)=\lambda \rho_{t}(X)$.

A4) If $X \in D, a \in L_{n}^{0}\left(\mathcal{F}_{t}\right)$ with $X+\bar{a} \in D$, then $\rho_{t}(X+\bar{a})=\rho_{t}(X)+\{-a\}$.

Remark 3.3 This definition is an obvious generalization of the definition in [9] to our dynamic setting.

- Property A4) allows to interpret $\rho($.$) as a monetary risk measure. Hence, an$ element $x_{t} \in \rho_{t}(X)$ can be understood as $n$-dimensional 'capital requirement' that can be set at time $t$ to hedge the risk of the financial position which has the final value $X$ at the maturity date $T$.

- Then, Property A3) is a straightforward dynamic version of the positive homogeneity property for risk measures.

- Property A1) is a monotonicity property consistent with $G_{T}$ : if the position $X$ is less risky than $Y$ with regard to the preorder $\geq_{G_{T}}$ then any 'capital requirement' $y_{t}$ which may be set at time $t$ to hedge the risk of $Y$ can also hedge the risk of $X$.

- As explained in [1], the subadditivity property A2), is a 'natural requirement' which can be 'stated in the brisk form a merger does not create extra risk'.

Remark 3.4 In general, we only have $\rho_{t}(\lambda X)=\lambda \rho_{t}(X)+\rho_{t}(0)$ if $\lambda$ is only known to be non-negative: $\lambda \in L_{1}^{0}\left(\mathbf{R}_{+}, \mathcal{F}_{t}\right)$. In particular

$$
\lambda \rho_{t}(X) \subseteq \rho_{t}(\lambda X) \quad \forall \lambda \in L_{1}^{0}\left(\mathbf{R}_{+}, \mathcal{F}_{t}\right) .
$$

Notice that the homogeneity property $\lambda \rho_{t}(X)=\rho_{t}(\lambda X)$ holds for any non negative multiplier $\lambda$ if and only if $\rho_{t}(0)=\{0\}$ which cannot be the case in our setting because of Axiom A4).

As stated in the following Proposition, the 'monotonicity property' $A 1$ can be replaced by the property $\mathrm{A} 1^{\prime}$ ) or by the property A1"). This shall be used later in some proofs. 
Proposition 3.5 A mapping $\rho$ defined on a convex $\mathcal{F}_{T}$-cone $D$ of $B_{G_{T}, n}$ with values on $\mathcal{S}_{[0, T]}^{n}$ is a $(d, n)$-coherent risk process if and only if it satisfies the statements $\left.\mathrm{A} 0\right)$ and $\mathrm{A} 2)$ - A4) together with: either

A1') If $X \in D$ is such that $X \geq_{G_{T}} 0$, then $\rho_{t}(0) \in \rho_{t}(X)$ for each $t \in[0, T]$.

or

A1") If $X \in D$ is such that $X \geq_{G_{T}} 0$, then $0 \in \rho_{t}(X)$ for each $t \in[0, T]$.

Proof. 1. It is obvious that A1) implies A1'). On the other hand, if A0) and A1') hold true, then $X \geq_{G_{T}} Y$ implies that $0 \in \rho_{t}(X-Y)$. Then, applying A2), we get $\rho_{t}(Y) \subseteq \rho_{t}(X)$.

2. Clearly the statements A0) together with $\left.\mathrm{A} 1^{\prime}\right)$ imply that a $(d, n)$-coherent risk process satisfies the statement A1"). To show the reciprocal, it suffices to apply A2).

The subadditivity Property A2) together with the positive homogeneity property A3) imply the convexity properties stated hereafter.

Proposition 3.6 Let $\rho$ be a $(d, n)$-coherent risk process defined on a convex $\mathcal{F}_{T}$-cone $D$ of $B_{G_{T}, n}$. Then, for each $X \in D$ and each $t \in[0, T], \rho_{t}(X)$ is a closed convex $\mathcal{F}_{t^{-}}$ measurable random set which satisfies: $\lambda_{t} \rho_{t}(X)+\left(1-\lambda_{t}\right) \rho_{t}(Y) \subset \rho_{t}\left(\lambda_{t} X+\left(1-\lambda_{t}\right) Y\right)$ for each $\mathcal{F}_{t}$-random variable $\lambda_{t}$ with values in $[0,1]$, and $X \in D$. Moreover, $\rho_{t}(0)$ is a closed convex $\mathcal{F}_{t}$-cone satisfying: $\rho_{t}(X)=\rho_{t}(X)+\rho_{t}(0)$ for all $X \in D$ and $t \leq T$.

We end this section with some continuity properties for the coherent risk measure processes.

Proposition 3.7 Let $\rho$ be a $(d, n)$-coherent risk process defined on a convex $\mathcal{F}_{T}$-cone $D \subseteq B_{G_{T}, n}$ containing $B_{G_{T}, n}\left(G_{T}, \mathcal{F}_{T}\right)$. Then the following claims hold true.

1. If $X, Y \in D$ are such that $X \leq_{G_{T}} Y$, then, for each $t \leq T, \rho_{t}(X)+\rho_{t}(0) \subseteq \rho_{t}(Y)$.

2. If $a \in L_{n}^{0}\left(\mathcal{F}_{t}\right)$ is such that $\bar{a} \in D$, then for each $s \in[t, T], \rho_{s}(\bar{a})=\rho_{s}(0)-\{a\}$.

3. If $X \in D$, and $a, b \in L_{n}^{0}\left(\mathcal{F}_{t}\right)$ are such that: $\bar{a}, \bar{b} \in D$ with $\bar{a} \leq_{G_{T}} X \leq_{G_{T}} \bar{b}$, then $\rho_{t}(0)+\{-a\} \subseteq \rho_{t}(X) \subseteq \rho_{t}(0)+\{-b\}$

4. For any $X, Y \in D$, we have

$$
\rho_{t}(Y)+\|X-Y\|_{G_{T}, n} \mathbf{1}_{d, n} \subseteq \rho_{t}(X) \subseteq \rho_{t}(Y)-\|X-Y\|_{G_{T}, n} \mathbf{1}_{d, n} \text { a.s. }
$$

\subsection{Measurable selectors of a risk-measure}

Let us first recall the concept of measurable selector of an $\mathcal{F}_{t^{-}}$-measurable random-set $\Gamma: \Omega \rightarrow \mathcal{P}\left(\mathbf{R}^{n}\right)$. A random variable $\gamma:\left(\Omega, \mathcal{F}_{t}, P\right) \rightarrow \mathbf{R}^{n}$ is called an $\mathcal{F}_{t}$-measurable selector of $\Gamma$ if $\gamma(\omega) \in \Gamma(\omega)$ for $P$ - a.e. $\omega$. Throughout this paper we make use of the expression 'by a measurable selection argument'. This expression refers to the measurable selection Theorem A.1 stated in Appendix. 
The concept of selectors turns out to be a useful tool for describing the random set. For instance, it is well known that a nonempty and closed-valued $\mathcal{F}_{t}$-random set is characterized by a countable set of measurable selectors $\left\{\gamma_{k}, k \in \mathbf{N}\right\}$ :

$$
\Gamma(\omega)=\operatorname{cl}\left[\left\{\gamma_{k}(\omega), k \in \mathbf{N}\right\}\right]
$$

The set $\left\{\gamma_{k}, k \in \mathbf{N}\right\}$ is commonly referred as a Castaing representation of $\Gamma$.

In the following we re-formulate important properties of a risk-measure process in terms of its selectors. Consider a $(d, n)$-coherent risk process $\rho$ defined on the convex $\mathcal{F}_{T}$-cone $D \subseteq B_{G_{T}, n}$ and some position $X \in D$. We denote by $\mathbf{S}_{\rho}(t, X)$ the set of all $\mathcal{F}_{t}$-measurable selectors of $\rho_{t}(X)$ :

$$
\mathbf{S}_{\rho}(t, X):=\left\{\gamma \in L_{n}^{0}\left(\mathcal{F}_{t}\right): \gamma \in \rho_{t}(X) P-a . s .\right\} .
$$

and by $\mathbf{S}_{\rho}^{\infty}(t, X)$ the set of bounded selectors

$$
\mathbf{S}_{\rho}^{\infty}(t, X):=\left\{\gamma \in \mathbf{S}_{\rho}(t, X): \bar{\gamma} \in B_{G_{T}, n}\left(\mathbf{R}^{d}, \mathcal{F}_{t}\right)\right\} .
$$

Definition 3.8 We say that a set-valued process $\rho$ from $D \subseteq E, E$ a metric space, into a metric space $F$ is continuous if it is both lower-semicontinuous and uppersemicontinuous in the following sense:

1.) $\rho$ is lower-semicontinuous at some point $X \in D$, if for all selectors $\left(Y_{t}\right)_{t \in[0, T]}$ of $\rho(X)$, i.e. $Y_{t} \in \mathbf{S}_{\rho}(t, X), \forall t$, and for any sequence $X^{m} \in D$ converging to $X$, there is a sequence $\left(Y_{t}^{m}\right)_{t \in[0, T]}$ of selectors of $\rho\left(X^{m}\right)$ such that $Y^{m} \rightarrow Y$ uniformly on $[0, T]$.

2.) $\rho$ is upper-semicontinuous at some $X \in D$, if for all $\varepsilon>0$ there is $\eta>0$ such that $\rho(X+B(0, \eta)) \subseteq \rho(X)+B(0, \varepsilon)$, i.e. any selector $Y$ of $\rho(X+B(0, \eta))$ can be written as the sum of a selector of $\rho(X)$ and a selector of $B(0, \varepsilon)$.

The continuity property (3.2) for a risk measure $\rho($.$) can be expressed as:$

Theorem $3.9 A(d, n)$-coherent risk process $\rho$ defined on a convex $\mathcal{F}_{T}$-cone $D \subseteq B_{G_{T}, n}$ containing $B_{G_{T}, n}\left(G_{T}, \mathcal{F}_{T}\right)$ is continuous.

Proof. Assume that $X^{m} \in D$ converges to $X$, i.e. $\left\|X^{m}-X\right\|_{G_{T}, n} \rightarrow 0$. Consider any selector $\xi:=\left(\xi_{t}\right)_{t \in[0, T]}$ of $\rho(X)$, i.e. such that $\xi_{t} \in \mathbf{S}_{\rho}(t, X)$ for any $t \in[0, T]$. From $\rho_{t}(X) \subseteq \rho_{t}\left(X^{m}\right)-\left\|X-X^{m}\right\|_{G_{T}, n} \mathbf{1}_{d, n}$ a.s. we deduce by a measurable selection argument the existence of $\xi_{t}^{m} \in \mathbf{S}_{\rho}\left(t, X^{m}\right)$ such that $\xi_{t}=\xi_{t}^{m}-\left\|X-X^{m}\right\|_{G_{T}, n} \mathbf{1}_{d, n}$ a.s. for all $m$. We then deduce that $\xi_{t}^{m}$ converges uniformly to $\xi$ on $[0, T]$.

From $\rho_{t}(Y)+\|X-Y\|_{G_{T}, n} \mathbf{1}_{d, n} \subseteq \rho_{t}(X)$ it is clear that $\rho$ is upper-semicontinuous.

As established in previous literature, the notion of risk measure is strongly related to the notion of acceptance set. This remains valid in our vector-valued and dynamic setting as stated in the next Subsection 3.3. Theorem (3.11) below is needed for the proofs of Subsection 3.3. They are analogous to Lemma 5.4.2 and Proposition 5.4.3 [10] which provide a characterization of closed subsets which are formed by the selectors of a closed random set. 
Definition 3.10 A set $\mathcal{A} \subseteq B_{G_{T}, n}$ is said $\mathcal{F}_{t^{-}}$-decomposable if for any countable partition $\left(\Omega_{t}^{i}\right)$ of $\Omega$ with $\Omega_{t}^{i} \in \mathcal{F}_{t}$ and any family $\left(X_{i}\right) \subseteq \mathcal{A}$, we have $\sum_{i} X_{i} \mathbf{1}_{\Omega_{t}^{i}} \in \mathcal{A}$ as soon as $\sum_{i} X_{i} \mathbf{1}_{\Omega_{t}^{i}} \in B_{G_{T}, n}$.

Theorem 3.11 Let $\mathcal{A}$ be a closed subset of $\left(B_{G_{T}, n}\left(\mathbf{R}^{d}, \mathcal{F}_{t}\right),\|\cdot\|_{G_{T}, n}\right)$. Then, $\mathcal{A}=$ $B_{G_{T}, n}\left(\Gamma, \mathcal{F}_{t}\right)$ for some set-valued $\mathcal{F}_{t}$-adapted mapping $\Gamma$ the values of which are closed sets if and only if $\mathcal{A}$ is $\mathcal{F}_{t}$-decomposable.

The proof is slightly different from [10] and is given in Appendix B.

\subsection{Acceptance set process}

Definition 3.12 A dynamic $(d, n)$-acceptance set process $\left(\mathcal{A}_{t}\right)_{t \in[0, T]}$ is a family of $\mathcal{F}_{t^{-}}$ convex cones of $B_{G_{T}, n}$ satisfying the following conditions:

B0) For any $t, \mathcal{A}_{t}$ is closed in $B_{G_{T}, n}$ endowed with $\|\cdot\|_{G_{T}, n}$.

B1) If $X \in B_{G_{T}, n}$ is such that $X \geq_{G_{T}} 0$, then $X \in \mathcal{A}_{t}, \forall t$.

B2) For each $t \leq T, B_{G_{T}, n}\left(\mathbf{R}^{n} \times 0_{d-n}, \mathcal{F}_{t}\right) \nsubseteq \mathcal{A}_{t}$.

B3) For each $t \leq T, \mathcal{A}_{t}$ is $\mathcal{F}_{t}$-decomposable.

Proposition 3.13 Let $\rho$ be a $(d, n)$-coherent risk process defined on a convex $\mathcal{F}_{T}$-cone $D \subseteq B_{G_{T}, n}$ containing $B_{G_{T}, n}\left(G_{T}, \mathcal{F}_{T}\right)$, and consider the set $\mathcal{A}^{\rho}=\left(\mathcal{A}_{t}^{\rho}\right)$ defined by

$$
\mathcal{A}_{t}^{\rho}:=\left\{X \in D: \rho_{t}(0) \subseteq \rho_{t}(X)\right\} .
$$

We suppose that $D$ is $\mathcal{F}_{T}$-decomposable and closed in $B_{G_{T}, n}$ endowed with $\|\cdot\|_{G_{T}, n}$. Then $\left(\mathcal{A}_{t}^{\rho}\right)$ is a dynamic $(d, n)$-acceptance set process, and can be also written as

$$
\mathcal{A}_{t}^{\rho}=\left\{X \in D: 0 \in \rho_{t}(X)\right\} .
$$

Proof. Statement B1) is immediate. Let us prove B2), i.e. $L^{0}\left(\mathbf{R}^{n} \times 0_{d-n}, \mathcal{F}_{t}\right) \nsubseteq \mathcal{A}_{t}^{\rho}$. By assumption there is an $\mathcal{F}_{t^{-}}$adapted non-null set $\Lambda_{t}$ such that $\rho_{t}(0) \neq \mathbf{R}^{n}$ on $\Lambda_{t}$. Recall that, by definition of random sets, the graph $\left\{(\omega, x) \in \Omega \times \mathbf{R}^{n}: x \in \rho_{t}(0)\right\}$ is $\mathcal{F}_{t} \times \mathcal{B}\left(\mathbf{R}^{n}\right)$-measurable. It follows that its complement is also $\mathcal{F}_{t} \times \mathcal{B}\left(\mathbf{R}^{n}\right)$-measurable, and of full measure on the state space $\Lambda_{t} \times \mathbf{R}^{n}$. By a measurable selection argument, we deduce the existence of $y_{t} \in L_{n}^{0}\left(\mathcal{F}_{t}\right)$ such that $y_{t} \notin \rho_{t}(0)$ on $\Lambda_{t}$. We may assume without loss of generality that $y_{t}=0$ on the complement of $\Lambda_{t}$ and dividing by $\left|y_{t}\right|+1$, we may assume that $y_{t} \in L_{n}^{\infty}\left(\mathcal{F}_{t}\right)$. Hence $\overline{y_{t}}:=\overline{y_{t}} I_{\Lambda_{t}} \in L_{d}^{\infty}\left(\mathbf{R}^{n} \times 0_{d-n}, \mathcal{F}_{t}\right)$. We claim that $\overline{y_{t}} \notin \mathcal{A}_{t}^{\rho}$. Indeed, in the contrary case, $y_{t} \in D$ and $\rho_{t}(0) \subseteq \rho_{t}\left(y_{t}\right)$ implies that $0 \in \rho_{t}(0) \subseteq \rho_{t}(0)+\left\{-y_{t}\right\}$. We deduce that $y_{t} \in \rho_{t}(0)$ hence a contradiction.

Let us show Statement B0). To do so, consider $X^{m} \rightarrow X$ where $X^{m} \in \mathcal{A}_{t}^{\rho}$. Since $D$ is closed, $X \in D$. Moreover, $X^{m}=\left(X^{m}-X\right)+X \leq_{G_{T}}\left\|X^{m}-X\right\|_{G_{T}, n} \mathbf{1}_{d, n}+X$ implies that $\rho_{t}\left(X^{m}\right) \subseteq \rho_{t}(X)-\left\|X^{m}-X\right\|_{G_{T}, n} \mathbf{1}_{d, n}$. Since $0 \in \rho_{t}\left(X^{m}\right)$ we deduce that $\left\|X^{m}-X\right\|_{G_{T}, n} \mathbf{1}_{d, n} \in \rho_{t}(X)$. As $\rho_{t}(X)$ is closed, we deduce that $0 \in \rho_{t}(X)$, i.e. $X \in \mathcal{A}_{t}^{\rho}$.

Let us show Statement B3). Consider a partition $\left(\Omega_{t}^{i}\right)$ of $\Omega$ with $\Omega_{t}^{i} \in \Omega$ and $\left(X^{i}\right) \subseteq \mathcal{A}_{t}^{\rho}$. Since $D$ is a $\mathcal{F}_{T}$-cone, $X \in D$ implies $X 1_{\Omega_{t}^{i}} \in D$. By virtue of (3.1), 
$1_{\Omega_{t}^{i}} \rho_{t}(X) \subseteq \rho_{t}\left(X 1_{\Omega_{t}^{i}}\right)$, hence $X 1_{\Omega_{t}^{i}} \in \mathcal{A}_{t}^{\rho}$ if $X \in \mathcal{A}_{t}^{\rho}$. From there, using Axiom A2), we deduce that $\sum_{i=1}^{n} X^{i} 1_{\Omega_{t}^{i}} \in \mathcal{A}_{t}^{\rho}$ for all $n$. Since $\sum_{i=1}^{\infty} X^{i} 1_{\Omega_{t}^{i}} \in D$, there exists $\kappa \geq 0$ such that $-\kappa \mathbf{1}_{d, n} \leq_{G_{T}} \sum_{i=1}^{\infty} X^{i} 1_{\Omega_{t}^{i}} \leq_{G_{T}} \kappa \mathbf{1}_{d, n}$ hence $-\kappa \mathbf{1}_{d, n} \leq_{G_{T}} X^{i} 1_{\Omega_{t}^{i}} \leq_{G_{T}} \kappa \mathbf{1}_{d, n}$ for all $i$. From the inequality

$$
\sum_{i=1}^{n}\left(X^{i}+\kappa \mathbf{1}_{d, n}\right) 1_{\Omega_{t}^{i}} \leq_{G_{T}} \sum_{i=1}^{\infty}\left(X^{i}+\kappa \mathbf{1}_{d, n}\right) 1_{\Omega_{t}^{i}}
$$

we deduce that

$$
0 \in \rho_{t}\left(\sum_{i=1}^{n} X^{i} 1_{\Omega_{t}^{i}}\right) \subseteq \rho_{t}\left(\sum_{i=1}^{\infty} X^{i} 1_{\Omega_{t}^{i}}\right)-\left(\sum_{i=n+1}^{\infty} 1_{\Omega_{t}^{i}}\right) \kappa \mathbf{1}_{d, n} .
$$

Making $n$ converged to $\infty$, we get that $0 \in \rho_{t}\left(\sum_{i=1}^{\infty} X^{i} 1_{\Omega_{t}^{i}}\right)$ so that $\sum_{i=1}^{\infty} X^{i} 1_{\Omega_{t}^{i}} \in \mathcal{A}_{t}^{\rho}$.

At last, $\mathcal{A}_{t}^{\rho}$ is an $\mathcal{F}_{t^{-}}$-cone. Indeed, if $X \in D$ with $0 \in \rho_{t}(X)$ and $\lambda \in L_{1}^{0}\left(\mathbf{R}^{+}, \mathcal{F}_{t}\right)$ is such that $\lambda X \in B_{G_{T}, n}$, then using Axiom A3) and property (3.2)

$$
\left.0 \in\left(\lambda+j^{-1}\right) \rho_{t}(X)=\rho_{t}\left(\left(\lambda+j^{-1}\right)\right) X\right) \subset \rho_{t}(\lambda X)-j^{-1}\|X\|_{G_{T}, n} \mathbf{1}_{d, n} .
$$

Making $j$ converged to 0 , we deduce that $0 \in \rho_{t}(\lambda X)$ so that $\lambda X \in \mathcal{A}_{t}$.

The following theorem shows the link between the notions of risk measure processes and acceptance set processes.

Theorem 3.14 Let $D$ be a convex $\mathcal{F}_{T}$-cone of $B_{G_{T}, n}$ which is $\mathcal{F}_{t}$-decomposable. Let $\mathcal{A}=\left(\mathcal{A}_{t}\right)$ be closed subsets of $\left(B_{G_{T}, n},\|\cdot\|_{G_{T}, n}\right)$. For each $X \in D$ define $\mathcal{L}^{\mathcal{A}}(t, X)$ by:

$$
\mathcal{L}^{\mathcal{A}}(t, X):=\left\{a \in L_{n}^{0}\left(\mathcal{F}_{t}\right): \bar{a}+X \in \mathcal{A}_{t}\right\}
$$

Then, $\mathcal{A}$ is a dynamic $(d, n)$-acceptance set process if and only if there exists some $(d, n)$-coherent risk process $\rho$ on $D \subset B_{G_{T}, n}$ such that

$$
\mathbf{S}_{\rho}^{\infty}(t, X)=\mathcal{L}^{\mathcal{A}}(t, X):=\left\{a \in L_{n}^{0}\left(\mathcal{F}_{t}\right): \bar{a}+X \in \mathcal{A}_{t}\right\}, \quad \forall X \in D
$$

Proof. We denote by $\overline{\mathcal{L}}^{\mathcal{A}}(t, X)$ the set of all $\bar{\gamma}$ such that $\gamma \in \mathcal{L}^{\mathcal{A}}(t, X)$.

Step 1. Assume that $\mathcal{A}$ is a dynamic $(d, n)$-acceptance process. We are going to show that $\mathbf{S}_{\rho}^{\infty}(t, X)=\mathcal{L}^{\mathcal{A}}(t, X)$ for some coherent risk process $\rho$.

Observe that $\overline{\mathcal{L}}^{\mathcal{A}}(t, X)$ is $\mathcal{F}_{t}$-decomposable and closed in $\left(B_{G_{t}, n},\|\cdot\|_{G_{T}, n}\right)$. By virtue of Theorem $3.11, \overline{\mathcal{L}}^{\mathcal{A}}(t, X)=B_{G_{T}, n}\left(\bar{\rho}_{t}(X), \mathcal{F}_{t}\right)$ where $\bar{\rho}_{t}(X)$ is a set-valued $\mathcal{F}_{t}$ measurable mapping from $\Omega \times[0, T]$ into $\mathcal{P}\left(\mathbf{R}^{d}\right)$. Moreover, $\bar{\rho}_{t}(X) \subseteq \mathbf{R}^{n} \times 0_{d-n}$ is a.s. closed. It follows that the projection $\rho_{t}(X) \subseteq \mathbf{R}^{n}$ of $\bar{\rho}_{t}(X)$ onto $\mathbf{R}^{n}$ is also a.s. closed and $\mathcal{L}^{\mathcal{A}}(t, X) \subseteq \mathbf{S}_{\rho}(t, X)$. Now, let us show that $\rho$ is a risk measure process. We start by proving Axiom 4$)$.

1. Axiom 4): We break the proof of Axiom A4) in several steps. 
step a. If $a \in L_{n}^{0}\left(\mathcal{F}_{t}\right)$ is s.t. $X$ and $X+\bar{a} \in D$, then

$$
\begin{aligned}
\mathcal{L}^{\mathcal{A}}(t, X+\bar{a}) & =\left\{x_{t} \in L_{n}^{0}\left(\mathcal{F}_{t}\right): \overline{x_{t}}+\bar{a}+X \in \mathcal{A}_{t}\right\} \\
& =-a+\left\{\left(x_{t}+a\right) \in L_{n}^{0}\left(\mathcal{F}_{t}\right): \overline{x_{t}+a}+X \in \mathcal{A}_{t}\right\} \\
& =-a+\mathcal{L}^{\mathcal{A}}(t, X) .
\end{aligned}
$$

Hence $B_{G_{T}, n}\left(\bar{\rho}_{t}(X+\bar{a}), \mathcal{F}_{t}\right)=-\bar{a}+B_{G_{T}, n}\left(\bar{\rho}_{t}(X), \mathcal{F}_{t}\right)$.

step b. If $X \in L_{d}^{0}\left(G_{T}, \mathcal{F}_{T}\right) \cap B_{G_{T}, n}$, then $X \in \mathcal{A}_{t}$. We deduce that $\mathcal{L}^{\mathcal{A}}(t, 0) \subseteq$ $\mathcal{L}^{\mathcal{A}}(t, X)$ and $0 \in \overline{\mathcal{L}}^{\mathcal{A}}(t, 0)=B_{G_{T}, n}\left(\bar{\rho}_{t}(0), \mathcal{F}_{t}\right) \subseteq B_{G_{T}, n}\left(\bar{\rho}_{t}(X), \mathcal{F}_{t}\right)$ a.s. In particular, $0 \in \bar{\rho}_{t}(X)$ a.s. More generally, if $X \in D$, then $X+\|X\|_{G_{T}, n} \mathbf{1}_{d, n} \geq_{G_{T}} 0$ a.s. so that $0 \in \bar{\rho}_{t}\left(X+\|X\|_{G_{T}, n} \mathbf{1}_{d, n}\right)$ a.s. Since $B_{G_{T}, n}\left(\bar{\rho}_{t}\left(X+\|X\|_{G_{T}, n} \mathbf{1}_{d, n}\right), \mathcal{F}_{t}\right)=-\|X\|_{G_{T}, n} \mathbf{1}_{d, n}+$ $B_{G_{T}, n}\left(\bar{\rho}_{t}^{\mathcal{A}}(X), \mathcal{F}_{t}\right)$, we deduce that $\|X\|_{G_{T}, n} \mathbf{1}_{d, n} \in B_{G_{T}, n}\left(\bar{\rho}_{t}(X), \mathcal{F}_{t}\right) \neq \emptyset$

step c. In this step we are going to prove that $\bar{\rho}_{t}(X+\bar{a})=-\bar{a}+\bar{\rho}_{t}(X)$ a.s. Notice that it is sufficient to verify the first inclusion $\bar{\rho}_{t}(X+\bar{a}) \subseteq-\bar{a}+\bar{\rho}_{t}(X)$, as the second one can be obtained by symmetry. Assume to the contrary that $\bar{\rho}_{t}(X+\bar{a}) \subseteq-\bar{a}+\bar{\rho}_{t}(X)$ does not hold. We construct by a measurable selection argument $\gamma_{t} \in L_{n}^{0}\left(\mathbf{R}^{n}, \mathcal{F}_{t}\right)$ such that $\gamma_{t} \in \bar{\rho}_{t}(X+\bar{a})$ and $\left|\gamma_{t}\right| \leq N, N$ large enough, on a non null set $\Lambda_{t}$ while $\gamma_{t} \notin-a+\bar{\rho}_{t}(X)$. Using step b., it is possible to choose $\gamma_{t}^{1}$ in the non-empty set $B_{G_{T}, n}\left(t, \bar{\rho}_{t}(X+\bar{a})\right)$. We get that $\gamma^{*}:=\gamma_{t} \mathbf{1}_{\Lambda_{t}}+\gamma_{t}^{1} \mathbf{1}_{\Omega \backslash \Lambda_{t}} \in B_{G_{T}, n}\left(t, \bar{\rho}_{t}(X+\bar{a})\right)=-\bar{a}+B_{G_{T}, n}\left(\bar{\rho}_{t}(X), \mathcal{F}_{t}\right)$ where the last equality follows from step a. This last equality yields to a contradiction. We conclude that Axiom A.4) holds true.

2. Axiom 0): By the Property B2) of acceptance set processes, it is possible to choose $y_{t} \in B_{G_{T}, n}\left(\mathbf{R}^{n}, \mathcal{F}_{t}\right)$ such that $\overline{y_{t}} \notin \mathcal{A}_{t}$, we obtain $\overline{y_{t}} \notin \overline{\mathcal{L}}^{\mathcal{A}}(t, 0)$. It follows that there exists a non null set $\Lambda_{t} \in \mathcal{F}_{t}$ s.t. $y_{t} \notin \rho_{t}(0)$ on $\Lambda_{t}$, i.e. $\rho_{t}(0)$ is not identically equal to $\mathbf{R}^{n}$. We then have proved Axiom A.0).

3. Axiom 1): By Property B1) of acceptance set processes, $X \in L_{d}^{0}\left(G_{T}, \mathcal{F}_{T}\right) \cap D$ implies that $X \in \mathcal{A}_{t}$. We deduce that $\mathcal{L}^{\mathcal{A}}(t, 0) \subseteq \mathcal{L}^{\mathcal{A}}(t, X)$ and $B_{G_{T}, n}\left(\bar{\rho}_{t}(0), \mathcal{F}_{t}\right) \subseteq$ $B_{G_{T}, n}\left(\bar{\rho}_{t}(X), \mathcal{F}_{t}\right)$. Arguing as previously, we get that $\rho_{t}(0) \subseteq \rho_{t}(X)$ a.s. Hence, Axiom A.1) holds.

4. Axiom 2) and Axiom3): These axioms follow directly from the fact that $\mathcal{A}_{t}$ is an $\mathcal{F}_{t}$-convex cone.

Step 2. Reciprocally, suppose that $\mathbf{S}_{\rho}^{\infty}(t, X)=\mathcal{L}^{\mathcal{A}}(t, X)$ for some $(d, n)$-coherent risk process $\rho$. We are going to show that $\mathcal{A}$ is a dynamic $(d, n)$-acceptance set process.

We verify first, that $\mathcal{A}_{t}$ is an $\mathcal{F}_{t}$-convex cone. Let us prove that it is stable under addition. If $X, Y \in \mathcal{A}_{t}$, then $0 \in \mathcal{L}^{\mathcal{A}}(t, X) \cap \mathcal{L}^{\mathcal{A}}(t, Y)$, i.e. $0 \in \rho_{t}(X) \cap \rho_{t}(Y)$ a.s., and from $\rho_{t}(X)+\rho_{t}(Y) \subseteq \rho_{t}(X+Y)$ we deduce that $0 \in \rho_{t}(X)+\rho_{t}(Y) \subseteq \rho_{t}(X+Y)$. Then $0 \in \mathcal{L}^{\mathcal{A}}(t, X+Y)$ and $X+Y \in \mathcal{A}_{t}$.

Let us consider $\lambda_{t} \in L^{0}\left(\mathbf{R}_{++}, \mathcal{F}_{t}\right)$ and $X \in \mathcal{A}_{t}$ such that $\lambda_{t} X \in B_{G_{T}, n}$. Then, $0=\lambda_{t} \times 0 \in \lambda_{t} \rho_{t}(X)=\rho_{t}\left(\lambda_{t} X\right)$. We then deduce that $0 \in \mathcal{L}^{\mathcal{A}}\left(t, \lambda_{t} X\right)$ hence $\lambda_{t} X \in \mathcal{A}_{t}$. If we only have $\lambda_{t} \in L^{0}\left(\mathbf{R}_{+}, \mathcal{F}_{t}\right)$, then $\left(\lambda_{t}+n^{-1}\right) X \in \mathcal{A}_{t}$ for all $n \geq 1$. Since, $\mathcal{A}_{t}$ is closed in $B_{G_{T}, n}$ by assumption, we conclude that $\lambda_{t} X \in \mathcal{A}_{t}$ as $n$ goes to $\infty$.

1. Axiom B0) This property holds true by assumption. 
2. Axiom B1) If $X \in B_{G_{T}, n}\left(G_{T}, \mathcal{F}_{t}\right)$ then, by assumption, $\rho_{t}(0) \subseteq \rho_{t}(X)$ a.s. so that $0 \in \rho_{t}(X)$. We deduce that $0 \in \mathcal{L}^{\mathcal{A}}(t, X)$ and $X \in \mathcal{A}_{t}$.

3. Axioms B2) and B3) These axioms can been shown by following the same arguments as in Proposition 3.13 .

\section{Dual representation of a risk process}

\subsection{General dual representation}

Recall that $B_{G_{T}, n}$ endowed with $\|\cdot\|_{G_{T}, n}$ is a Banach space. We denote by $b a_{G_{T}, n}$ the topological dual space of $B_{G_{T}, n}$. We define the set of positive linear forms

$$
b a_{G_{T}, n}^{+}:=\left\{\varphi \in b a_{G_{T}, n}: \varphi(X) \geq 0 \text { for all } X \geq_{G_{T}} 0\right\} .
$$

Definition 4.1 We say that a subset $\Lambda$ of $b a_{G_{T}, n}$ is $\mathcal{F}_{t}$-stable if for all $\lambda \in L^{\infty}\left(\mathbf{R}_{+}, \mathcal{F}_{t}\right)$ and $\varphi \in \Lambda$, the linear form $\varphi^{\lambda}: X \ni B_{G_{T}, n} \mapsto \varphi(\lambda X)$, belongs to $\Lambda$.

We state hereafter the main result of this section: the dual characterization of coherent risk processes.

Theorem 4.2 (Dual characterization) Let $\rho$ be a $\mathcal{S}_{[0, T]}^{n}$-valued mapping on a $\mathcal{F}_{T^{-}}$ cone $D \subseteq B_{G_{T}, n}$. Assume that $D$ is closed and contains $B_{G_{T}, n}\left(G_{T}, \mathcal{F}_{T}\right)$. Then, the following statements are equivalent:

(i) $\rho$ is a $(d, n)$-coherent risk process on $D$.

(ii) There exists a $\sigma\left(b a_{G_{T}, n}, B_{G_{T}, n}\right)$-closed subset $\mathcal{P}_{b a}(t) \neq\{0\}$ of $b a_{G_{T}, n}^{+}$which is $\mathcal{F}_{t}$-stable and satisfies the equality

$$
\mathbf{S}_{\rho}^{\infty}(t, X)=\left\{x_{t} \in B_{G_{T}, n}\left(\mathbf{R}^{n}, \mathcal{F}_{t}\right): \varphi\left(X+\overline{x_{t}}\right) \geq 0, \forall \varphi \in \mathcal{P}_{b a}(t)\right\}
$$

Proof.

1. Let us prove (i) $\Rightarrow$ (ii). To do so, consider $C_{t}:=\left\{X \in D: 0 \in \rho_{t}(X)\right\}$. Observe that $B_{G_{T}, n}\left(G_{T}, \mathcal{F}_{T}\right) \subseteq C_{t}$. It follows that the positive dual

$$
\mathcal{P}_{b a}(t):=\left\{\varphi \in b a_{G_{T}, n}: \varphi(X) \geq 0, \forall X \in C_{t}\right\}
$$

is contained in $b a_{G_{T}, n}^{+}$and is obviously $\sigma\left(b a_{G_{T}, n}, B_{G_{T}, n}\right)$-closed. By virtue of the continuity property (3.2) of $\rho, C_{t}$ is closed in $B_{G_{T}, n}$. We can also easily check that it is a non-empty convex set. It follows that $C_{t}$ is the dual of $\mathcal{P}_{b a}(t)$. From there and from Proposition 3.13 and Theorem 3.14

$$
\begin{aligned}
\mathbf{S}_{\rho}^{\infty}(t, X) & =\left\{x_{t} \in L_{n}^{0}\left(\mathcal{F}_{t}\right): 0 \in \rho_{t}\left(X+\overline{x_{t}}\right) \text { a.s. }\right\}, \\
& =\left\{x_{t} \in L_{n}^{0}\left(\mathcal{F}_{t}\right): X+\overline{x_{t}} \in C_{t}\right\} \\
& =\left\{x_{t} \in L_{n}^{0}\left(\mathcal{F}_{t}\right): \varphi\left(X+\overline{x_{t}}\right) \geq 0, \forall \varphi \in \mathcal{P}_{b a}(t)\right\}
\end{aligned}
$$


Note that $\mathcal{P}_{b a}(t) \neq\{0\}$; otherwise $\rho_{t}(0)=\mathbf{R}^{n}$ which contradicts Axiom A0).

Since $C_{t}$ is stable under positive and bounded $\mathcal{F}_{t}$-measurable multiplication, we infer that $\mathcal{P}_{b a}(t)$ is $\mathcal{F}_{t}$-stable.

2. Let us show that (ii) $\Rightarrow$ (i).

Axiom A0). From the dual representation (4.3), we immediately deduce that $\mathbf{S}_{\rho}^{\infty}(t, X)$ is $B_{G_{T}, n}$-closed and decomposable since $\mathcal{P}_{b a}(t)$ is $\mathcal{F}_{t}$-stable. It follows from Theorem 3.11 that $\rho_{t}(X)$ is a.s. closed. It is clear from (4.3) that $0 \in \mathbf{S}_{\rho}^{\infty}(t, 0)$, hence $0 \in \rho_{t}(0)$ a.s. Now, we claim that $\rho_{t}(0) \neq \mathbf{R}^{n}$. Assume to the contrary that $\rho_{t}(0)=\mathbf{R}^{n}$, it follows that $\mathbf{S}_{\rho}^{\infty}(t, 0)=L^{\infty}\left(\mathbf{R}^{n}, \mathcal{F}_{t}\right)$ and from (4.3) we get that $\varphi\left(\mathbf{1}_{d, n}\right)=$ 0. As $\mathcal{P}_{b a}(t) \subseteq b a_{G_{T}, n}^{+}$we conclude from Lemma C.5 that $\mathcal{P}_{b a}(t)=\{0\}$, which is a contradiction to (ii).

Axiom A1), A2) and A4). It is easy to verify from (4.3) that the mapping $L_{\rho}^{\infty}(t, \cdot)$ satisfies the following properties

$$
\begin{array}{ll}
\text { a1 : } & X \geq_{G_{T}} Y \text { implies } L_{\rho}^{\infty}(t, Y) \subseteq L_{\rho}^{\infty}(t, X) \\
\text { a2 : } & L_{\rho}^{\infty}(t, X)+L_{\rho}^{\infty}(t, Y) \subseteq L_{\rho}^{\infty}(t, X+Y) \\
\text { a4 : } & a \in L_{n}^{0}\left(\mathbf{R}^{n}, \mathcal{F}_{t}\right) \text { with } X+\bar{a} \in D \text { implies } L_{\rho}^{\infty}(t, X+\bar{a})=L_{\rho}^{\infty}(t, X)-\{a\}
\end{array}
$$

We then conclude by using Lemma A.2 that $\rho$ satisfies axioms A1), A2) and A4). Notice that, as a consequence of these axioms, $\rho$ satisfies the continuity property (3.2).

Axiom A3). We have to show: if $\lambda \in L_{1}^{0}\left(\mathbf{R}_{++}, \mathcal{F}_{t}\right)$ is such that $\lambda X \in D$ for some $X \in D$, then $L_{\rho}^{\infty}(t, \lambda X)=\lambda L_{\rho}^{\infty}(t, X)$. Let us first consider $\lambda \in L^{\infty}\left(\mathbf{R}_{++}, \mathcal{F}_{t}\right)$ and denote $\lambda^{n}:=\lambda+n^{-1} \in L^{\infty}\left(\mathbf{R}_{++}, \mathcal{F}_{t}\right)$. Since $\mathcal{P}_{b a}(t)$ is $\mathcal{F}_{t^{-}}$stable, for all $n, \varphi^{\lambda^{n}}$ and $\varphi^{1 / \lambda_{n}}$ belong to $\mathcal{P}_{b a}(t)$. Therefore for all $x_{t} \in L_{\rho}^{\infty}(t, X), \varphi \in \mathcal{P}_{b a}(t)$, we have $\varphi^{\lambda^{n}}\left(X+\bar{x}_{t}\right)=\varphi\left(\lambda^{n} X+\lambda^{n} \bar{x}_{t}\right) \geq 0$. That is, $\lambda^{n} x_{t} \in L_{\rho}^{\infty}\left(t, \lambda^{n} X\right)$ and we get the inclusion $\lambda^{n} L_{\rho}^{\infty}(t, X) \subseteq L_{\rho}^{\infty}\left(t, \lambda^{n} X\right)$. The second inclusion is obtained similarly by considering $\varphi^{1 / \lambda^{n}}$. Now using the Lemma A.2, we obtain that: $\rho_{t}\left(\lambda^{n} X\right)=\lambda^{n} \rho_{t}(X)$. Letting $n$ go to $\infty$, by virtue of the continuity property (3.2), we get that: $\rho_{t}(\lambda X)=\lambda \rho_{t}(X)$.

The general case $\lambda \in L_{1}^{0}\left(\mathbf{R}_{++}, \mathcal{F}_{t}\right)$ is deduced from the above equality as follows:

$$
\rho_{t}\left(\frac{\lambda}{1+\lambda} Y\right)=\frac{\lambda}{1+\lambda} \rho_{t}(Y)=\lambda \rho_{t}\left(\frac{Y}{1+\lambda}\right), \quad \forall Y \in D .
$$

Taking $Y:=(1+\lambda) X \in D$ we conclude that $\rho_{t}(\lambda X)=\lambda \rho_{t}(X)$.

\subsection{Dual representation under Fatou property}

Definition 4.3A $\mathcal{S}_{[0, T]}^{n}$-valued mapping $\rho$ on $B_{G_{T}, n}\left(\mathbf{R}^{d}, \mathcal{F}_{T}\right)$ is said to satisfy the Fatou property if for all $X \in B_{G_{T}, n}$,

$$
\liminf _{m \rightarrow \infty} L_{\rho}^{\infty}\left(t, X^{m}\right) \subseteq L_{\rho}^{\infty}(t, X), \forall t
$$

for any bounded sequence $\left(X^{m}\right)$ in $B_{G_{T}, n}$ which converges to $X$ in probability. 
Remark 4.4 In the literature, a bounded sequence $\left(X^{m}\right)$ in $B_{G_{T}, n}$ which converges to $X$ in probability is said Fatou-convergent to $X$. This is an important convergence tool in arbitrage theory with friction.

The main result of this subsection states a dual representation of coherent risk processes satisfying the Fatou property. This dual representation is based on a duality between $B_{G_{T}, n}$ and the space $L_{d}^{1, n}\left(\mathbf{R}^{d}, \mathcal{F}_{T}\right)$ defined below, analogous to the duality between $L^{\infty}$ and $L^{1}$. This duality holds under the Conditions $\mathbf{g 4 - g 5}$ below. We assume throughout this subsection that:

g4: $\quad \mathbf{R}_{+}^{d} \backslash\{0\} \subseteq \operatorname{int}\left[G_{T}\right]$ or equivalently $G_{T}^{*} \backslash\{0\} \subseteq \operatorname{int} R_{+}^{d}$.

g5: $G_{T}^{*}$ and $G_{T}$ are both generated by a finite number of linearly independent and bounded generators denoted respectively by $\left(\xi_{i}^{*}\right)_{1 \leq i \leq N^{*}}$ and $\left(\xi_{i}\right)_{1 \leq i \leq N}$.

Remark 4.5 Notice that g4 and g5 are usual assumptions in the financial models of the literature.

Given the Conditions g4-g5, we consider the measurable decomposition:

$$
\mathbf{1}_{d, n}=\sum_{i=1}^{N} \alpha_{i} \xi_{i}, \quad \text { where } \alpha_{i} \in L_{d}^{0}\left(\mathbf{R}_{+}, \mathcal{F}_{T}\right), i=1, \cdots, N .
$$

For a random set $A \subseteq \mathbf{R}^{d}$, let $L_{d}^{1, n}\left(A, \mathcal{F}_{T}\right)$ be the set defined by:

$$
L_{d}^{1, n}\left(A, \mathcal{F}_{T}\right):=\left\{Z \in L_{d}^{0}\left(A, \mathcal{F}_{T}\right): Z \cdot\left(\alpha^{i} \xi^{i}\right) \in L_{d}^{1} \forall i=1, \cdots, N\right\} .
$$

Remark 4.6 In the case where $A=G_{T}^{*}$, the set $L_{d}^{1, n}\left(G_{T}^{*}, \mathcal{F}_{T}\right)$ is also equal to

$$
L_{d}^{1, n}\left(G_{T}^{*}, \mathcal{F}_{T}\right)=\left\{Z \in L_{d}^{0}\left(G_{T}^{*}, \mathcal{F}_{T}\right): Z \cdot \mathbf{1}_{d, n} \in L_{d}^{1}\right\}
$$

This is stated in Appendix C.

We denote by $\|\cdot\|_{d, n}$ the dual norm on $b a_{G_{T}, n}$. Notice that, for $Z \in L_{d}^{1, n}\left(G_{T}^{*}, \mathcal{F}_{T}\right)$, the linear form $\phi_{Z}:=X \mapsto E[Z X]$, belongs to $b a_{G_{T}, n}^{+}$. We shall denote $\|Z\|_{d, n}:=\left\|\phi_{Z}\right\|_{d, n}$.

The main result of this subsection, Theorem 4.8, relies on the characterization:

Proposition 4.7 Let $C$ be a decomposable convex set of $B_{G_{T}, n}$. The set $C$ is weak * closed (i.e. $\sigma\left(B_{G_{T}, n}, L_{d}^{1, n}\left(\mathbf{R}^{d}, \mathcal{F}_{T}\right)\right)$ - closed) if and only if $C \cap\left\{\xi:\|\xi\|_{G_{T}, n} \leq M\right\}$ is closed in probability $\forall M \in \mathbf{R}_{+}$.

The proof of this essential characterization result is provided in Appendix C. 
Theorem 4.8 Let $\rho$ be $a(d, n)$-coherent risk process on $B_{G_{T}, n}$. Assume that Conditions g1 - g5 hold. The following statements are equivalent:

(i) For each $t \in[0, T]$, there exists a closed $\mathcal{F}_{t}$-cone, $\{0\} \neq \mathcal{P}_{b a}(t) \subseteq\left(L_{d}^{1, n}\left(G_{T}^{*}, \mathcal{F}_{T}\right),\|\cdot\|_{d, n}\right)$ such that

$$
\mathbf{S}_{\rho}^{\infty}(t, X)=\left\{x_{t}: \bar{x}_{t} \in B_{G_{T}, n}, \varphi_{Z}\left(X+\overline{x_{t}}\right) \geq 0, \forall Z \in \mathcal{P}_{b a}(t)\right\}
$$

(ii) $\rho$ satisfies the Fatou property.

(iii) $C_{t}:=\left\{X \in B_{G_{T}, n}: \rho_{t}(0) \subset \rho_{t}(X)\right\}$ is $\sigma\left(B_{G_{T}, n}, L_{d}^{1, n}\left(\mathbf{R}^{d}, \mathcal{F}_{T}\right)\right)$-closed.

Proof.

1. We start by proving that (i) $\Rightarrow$ (ii). Let $\left(X^{m}\right)$ be a bounded sequence in $B_{G_{T}, n}$ which converges to $X$ in probability. Consider $x_{t} \in \liminf _{m \rightarrow \infty} L_{\rho}^{\infty}\left(t, X^{m}\right)$, let us show that $x_{t} \in L_{\rho}^{\infty}(t, X)$. Since $x_{t} \in L_{\rho}^{\infty}\left(t, X^{m}\right)$ for $m$ large enough, the dual representation (4.5) implies that $E Z_{T}\left(X^{m}+\overline{x_{t}}\right) \geq 0, \forall Z_{T} \in \mathcal{P}_{b a}(t)$. Observe that $\left(-Z_{T} X^{m}\right)$ is uniformly bounded from below by $-\kappa Z_{T} \mathbf{1}_{d, n}$ where $\kappa=\sup _{m}\left\|X^{m}\right\|_{G_{T}, n}$. Applying Fatou's lemma, we deduce that $E Z_{T}\left(X+\overline{x_{t}}\right) \geq 0, \forall Z_{T} \in \mathcal{P}_{b a}(t)$, i.e. $x_{t} \in L_{\rho}^{\infty}(t, X)$.

2. Let us show that (ii) $\Rightarrow$ (iii). Applying Proposition 4.7, it is sufficient to prove that $C_{t} \cap\left\{\xi:\|\xi\|_{G_{T}, n} \leq M\right\}$ is closed in probability whatever $M \in \mathbf{R}_{+}$. This is a direct consequence of (ii).

3. Observe that $\varphi_{Z} \geq 0$ iff $Z \in L_{d}^{1, n}\left(G_{T}^{*}, \mathcal{F}_{T}\right)$. Then, the implication $($ iii $) \Rightarrow(i)$ is obtained through the same arguments as the proof of (i) $\Rightarrow$ (ii) of Theorem 4.2.

Notice that in the case $d=n$ and $Z \in L_{d}^{1, n}\left(G_{T}^{*}, \mathcal{F}_{T}\right),\|Z\|_{d, n}$ is the usual norm on $L_{d}^{1}\left(\mathbf{R}^{d}, \mathcal{F}_{T}\right)$ associated with the norm $|x|:=x . \mathbf{1}$ on $\mathbf{R}^{d}$.

\section{Examples}

\subsection{Worst conditional expectation}

For each $t \in[0, T]$, let $\mathcal{A}_{t}^{\text {wce }}$ be the subset of $B_{G_{T}, n}$ defined by

$$
\mathcal{A}_{t}^{w c e}:=\left\{X \in B_{G_{T}, n}: E\left[X \cdot \xi_{i}^{\star} \mid \mathcal{F}_{t}\right] \geq 0 \quad \forall i=1, \cdots N^{\star}\right\} .
$$

We can easily verify that $\left(\mathcal{A}^{w c e}\right)_{t \in[0, T]}$ is a $(d, n)$-acceptance process. By virtue of Proposition (3.13), we can associate with the acceptance process $\left(\mathcal{A}_{t}^{w c e}\right)_{t \in[0, T]}$ a $(d, n)$ coherent risk measure process $\left(\rho_{t}^{w c e}\right)_{t \in[0, T]}$ whose measurable selectors are given by

$$
\begin{aligned}
\mathbf{S}_{\rho^{w c e}}^{\infty}(t, X) & =\left\{a \in L_{n}^{0}\left(\mathbf{R}^{n}, \mathcal{F}_{t}\right): E\left[(\bar{a}+X) \cdot \xi_{i}^{\star} \mid \mathcal{F}_{t}\right] \geq 0 \quad \forall i=1, \cdots, N^{\star}\right\} \\
& =\left\{a \in L_{n}^{0}\left(\mathbf{R}^{n}, \mathcal{F}_{t}\right): \bar{a} \cdot E\left[\xi_{i}^{\star} \mid \mathcal{F}_{t}\right]+E\left[X \cdot \xi_{i}^{\star} \mid \mathcal{F}_{t}\right] \geq 0 \quad \forall i=1, \cdots, N^{\star}\right\}
\end{aligned}
$$

This is the 'dynamic' version of the Worst Conditional Expectation (WCE) vectorvalued risk measure introduced in [9]. 


\subsection{Super-replication}

Let us consider the financial market of Example 2.1. As in the setting of [3], consider $\mathcal{Y}_{t, 0}^{T}$ the set of all admissible predictable portfolio processes of bounded variations expressed in physical units. Let $X$ be an European contingent claim $X \in B_{G_{T}, n}$. Let $D_{t}(X)$ be the set of all hedging prices of $X$ which is defined by

$$
D_{t}(X):=\left\{x_{t}: \bar{x}_{t} \in B_{G_{T}, n}\left(\mathbf{R}^{n} \times 0_{d-n}, \mathcal{F}_{t}\right): \exists Y \in \mathcal{Y}_{t, 0}^{T}, \bar{x}_{t}+Y_{T} \geq_{G_{T}}-X\right\} .
$$

It has been shown, see for example [10], that under the condition of existence of a strictly consistent price system, the following dual representation result holds true

$$
\begin{aligned}
D_{t}(X) & =\left\{x_{t}: \bar{x}_{t} \in B_{G_{T}, n}\left(\mathbf{R}^{n} \times 0_{d-n}, \mathcal{F}_{t}\right): Z_{t} \bar{x}_{t} \geq-E\left[Z_{T} X \mid \mathcal{F}_{t}\right], \forall Z \in \mathcal{M}_{t}^{T}\left(G^{*}\right)\right\}, \\
& =\left\{x_{t}: \bar{x}_{t} \in B_{G_{T}, n}\left(\mathbf{R}^{n} \times 0_{d-n}, \mathcal{F}_{t}\right): \varphi_{Z_{T}}\left(X+\bar{x}_{t}\right) \geq 0, \forall Z \in \mathcal{M}_{t}^{T}\left(G^{*}\right)\right\}
\end{aligned}
$$

where $\mathcal{M}_{t}^{T}\left(G^{*}\right)$ is the set of all martingales $\left(Z_{u}\right)_{u \in[t, T]}$ such that $Z_{u} \in G_{u}^{*} \forall u \geq t$. By Theorem 4.2, $D_{t}(X)=L_{\rho}^{\infty}(X, t)$ for some $(d, n)$-coherent risk process $\rho$. Moreover, if conditions $\mathbf{g} \mathbf{4}, \mathbf{g} \mathbf{5}$ are satisfied, then the risk measure $\rho$ satisfies the Fatou property. This is, for instance, the case if $d=2$.

Definition 5.1 $A(d, n)$-coherent risk process $\rho$ on $D=B_{G_{T}, n}$ is said weakly consistent in time if for any $s \leq t \leq T$ and $X \in B_{G_{T}, n}, 0 \in \rho_{t}(X)$ implies $0 \in \rho_{s}(X)$.

We easily deduce the following result:

Proposition 5.2 Let $\rho$ be a $(d, n)$-coherent risk process defined on $D=B_{G_{T}, n}$. The following statements are equivalent:

(i) The $(d, n)$-coherent risk process $\rho$ on $D=B_{G_{T}, n}$ is weakly consistent in time.

(ii) $\mathcal{P}_{b a}(s) \subseteq \mathcal{P}_{b a}(t)$ for all $s \leq t \leq T$.

In our example, $\mathcal{P}_{b a}(t)=\left\{Z_{T}: Z \in \mathcal{M}_{t}^{T}\left(G^{*}\right)\right\}$. It is clear that the property (ii) holds so that $\rho$ is weakly consistent in time.

\section{Appendices}

\section{A Auxiliary results}

For the convenience of the reader, we recall from [10] the measurable selection theorem.

Theorem A.1 Let $(\Omega, \mathcal{F}, P)$ be a complete probability space, let $(E, \mathcal{E})$ be a borel space and let $\Gamma \subseteq \Omega \times E$ be an element of the $\sigma$-algebra $\mathcal{F} \otimes \mathcal{E}$. Then the projection $\operatorname{Pr}_{\Omega}(\Gamma)$ of $\Gamma$ onto $\Omega$ is an element of $\mathcal{F}$, and there exists an E-valued random variable $\xi$ such that $\xi(\omega) \in \Gamma_{\omega}$ for all non-empty $\omega$-sections $\Gamma_{\omega}$ of $\Gamma$. 
Lemma A.2 Let $\mathcal{H} \subseteq \mathcal{F}$ be a $\sigma$-algebra. Suppose that the inclusion

$$
B_{G_{T}, n}\left(\Lambda_{1}, \mathcal{H}\right)+B_{G_{T}, n}\left(\Lambda_{2}, \mathcal{H}\right) \subseteq B_{G_{T}, n}\left(\Lambda_{3}, \mathcal{H}\right)
$$

holds for some $\mathcal{H} \times \mathcal{B}\left(\mathbf{R}^{d}\right)$-measurable set-valued mappings $\Lambda_{i}$. Then, the inclusion $\Lambda_{1}+\Lambda_{2} \subseteq \Lambda_{3}$ holds a.s.

Proof. We may assume without loss of generality that $B_{G_{T}, n}\left(\Lambda_{1}, \mathcal{H}\right) \neq \emptyset$ and $B_{G_{T}, n}\left(\Lambda_{2}, \mathcal{H}\right) \neq \emptyset$. Suppose by contradiction that the inclusion $\Lambda_{1}+\Lambda_{2} \subseteq \Lambda_{3}$ fails on a non-null set $B \in \mathcal{H}$. We deduce by a measurable selection argument $\xi_{1} \in L_{d}^{0}\left(\mathbf{R}^{d}, \mathcal{H}\right)$ and $\xi_{2} \in L_{d}^{0}\left(\mathbf{R}^{d}, \mathcal{H}\right)$ such that $\xi_{1} \in \Lambda_{1}$ and $\xi_{2} \in \Lambda_{2}$ while $\xi_{1}+\xi_{2} \notin \Lambda_{3}$ on the set $B$. We may assume without loss of generality that $\xi_{1}$ and $\xi_{2}$ are bounded. Taking $\hat{\xi}_{1} \in B_{G_{T}, n}\left(\Lambda_{1}, \mathcal{H}\right)$ and $\hat{\xi}_{2} \in B_{G_{T}, n}\left(\Lambda_{2}, \mathcal{H}\right)$, we set $\tilde{\xi}_{i}:=\xi_{i} \mathbf{1}_{B}+\hat{\xi}_{i} \mathbf{1}_{B^{c}}, i=1,2$. It is immediate that $\tilde{\xi}_{i} \in B_{G_{T}, n}\left(\Lambda_{i}, \mathcal{H}\right)$. Using the hypothesis of the lemma, it follows that $\tilde{\xi}_{1}+\tilde{\xi}_{2} \in B_{G_{T}, n}\left(\Lambda_{3}, \mathcal{H}\right)$ hence a contradiction.

Lemma A.3 Let $\left(\xi^{k}\right) \subset B_{G_{T}, n}\left(\mathbf{R}^{d}, \mathcal{F}_{t}\right)$ be a countable family. For each $\omega \in \Omega$, let $\Gamma(\omega)$ be the closure in $\mathbf{R}^{d}$ of the set $\left\{\xi_{k}(\omega)\right\}$. Then, $B_{G_{T}, n}\left(\Gamma, \mathcal{F}_{t}\right)$ is the closure in $\left(B_{G_{T}, n},\|\cdot\|_{G_{T}, n}\right)$ of a countable set of random variables in $B_{G_{T}, n}\left(\mathbf{R}^{d}, \mathcal{F}_{t}\right)$ of the form $\sum_{k=1}^{\infty} \mathbf{1}_{\Omega_{t}^{k}} \xi^{k}$ where $\left(\Omega_{t}^{k}\right)$ are $\mathcal{F}_{t}$-measurable partitions of $\Omega$.

Proof. Observe that

$$
\{(\omega, x): x \in \Gamma(\omega)\}=\bigcap_{q \in \mathbf{Q}^{+}} \bigcup_{k}\left\{\left|x-\xi_{k}(\omega)\right|<q\right\}
$$

where $\mathbf{Q}^{+}$is the set of all strictly positive rational number. It follows that the set-valued mapping $\omega \mapsto \Gamma(\omega)$ is $\mathcal{F}_{t^{-}}$adapted. Let us consider $\xi \in B_{G_{T}, n}\left(\Gamma, \mathcal{F}_{t}\right)$. Then, a.s $(\omega)$, for any $q \in \mathbf{Q}^{+}$, there exists $k$ such that $-q \mathbf{1}_{d, n} \leq_{\mathbf{R}_{+}^{d}} \xi(\omega)-\xi^{k}(\omega) \leq_{\mathbf{R}_{+}^{d}} q \mathbf{1}_{d, n}$, i.e. $\Omega=\cup_{k} B_{k}^{q}$ where $B_{k}^{q}=\left\{\omega:-q \mathbf{1}_{d, n} \leq_{\mathbf{R}_{+}^{d}} \xi(\omega)-\xi^{k}(\omega) \leq_{\mathbf{R}_{+}^{d}} q \mathbf{1}_{d, n}\right\} \in \mathcal{F}_{t}$. We put $\Omega_{t}^{1}=B_{1}$ and $\Omega_{t}^{k}=B_{k} \backslash\left(\cup_{j \leq k-1} \Omega_{t}^{j}\right)$. It is then straightforward that $\left\|\xi-\sum_{k} \xi^{k} \mathbf{1}_{\Omega_{t}^{k}}\right\|_{G_{T}, n} \leq q$.

\section{B Proof of Theorem 3.11}

Let $\mathcal{A}$ be a closed subset of $\left(B_{G_{T}, n}\left(\mathbf{R}^{d}, \mathcal{F}_{t}\right),\|\cdot\|_{G_{T}, n}\right)$. It is clear that $\mathcal{A}=B_{G_{T}, n}\left(\Gamma, \mathcal{F}_{t}\right)$ is $\mathcal{F}_{t}$-decomposable.

Reciprocally, suppose that $\mathcal{A}$ is $\mathcal{F}_{t^{-}}$-decomposable. Consider a countable dense subset $\left(x_{i}\right)$ of $\mathbf{R}^{d}$ and let us define $a_{i}:=\inf _{\gamma \in \mathcal{A}} E\left|\gamma-x_{i}\right| \wedge 1$. Then, there exists $\gamma_{i, j} \in \mathcal{A}$ such that $E\left|\gamma_{i, j}-x_{i}\right| \wedge 1 \leq a_{i}+j^{-1}$ for any $j \in \mathbf{N}$. Let us define $\Gamma(\omega)$ as the closure of $\left(\gamma_{i, j}(\omega)\right)$ in $\mathbf{R}^{d}$. By virtue of Lemma A.3, $B_{G_{T}, n}\left(\Gamma, \mathcal{F}_{t}\right) \subseteq \mathcal{A}$ if $\mathcal{A}$ is closed and decomposable. Under this condition, suppose that there exists $\xi \in \mathcal{A}$ such that $\xi \notin B_{G_{T}, n}\left(\Gamma, \mathcal{F}_{t}\right)$. Then, it suffices to repeat the reasoning of Proposition 5.4.3 [10] with $p=0$. 
Remark B.1 Observe that if $\mathcal{A}=B_{G_{T}, n}\left(\Gamma, \mathcal{F}_{t}\right)$ and $\mathcal{A}$ is a closed subset of $B_{G_{T}, n}\left(\mathbf{R}^{d}, \mathcal{F}_{t}\right)$, then necessarily $\Gamma$ is closed. Indeed, consider the case where $0 \in \Gamma$. If $\Gamma$ is not closed, we may find a selector $\gamma \in L^{\infty}\left(\bar{\Gamma}, \mathcal{F}_{t}\right)$ such that $\gamma \notin \Gamma$ on a non null set. Again by a measurable selection argument, we may construct a sequence $\gamma_{m} \in L^{0}\left(\Gamma, \mathcal{F}_{t}\right), m \in \mathbf{N}$, such that $\left|\gamma-\gamma^{m}\right| \leq m^{-1}$ so that we have $\gamma_{m} \in B_{G_{T}, n}\left(\Gamma, \mathcal{F}_{t}\right)=\mathcal{A}$ and $\gamma^{m} \rightarrow \gamma$. We then get a contradiction since $\mathcal{A}$ is closed.

\section{Proof of Proposition 4.7}

For our dual-characterization theorem, we use the duality between the Banach space $\left(B_{G_{T}, n},\|\cdot\|_{G_{T}, n}\right)$ and the vector space $L_{d}^{1}\left(\mathbf{R}^{d}, \mathcal{F}_{t}\right)$ equipped with a suitable norm. It turns out that under the supplementary conditions $\mathbf{g} \mathbf{4}-\mathbf{g} \mathbf{5}, L_{d}^{1}\left(\mathbf{R}^{d}, \mathcal{F}_{t}\right)$ is equal to the subspace Vect $L_{d}^{1}\left(G_{T}^{*}, \mathcal{F}_{T}\right)$ of the Banach space $b a_{G_{T}, n}$. The proof is based on properties stated in the following paragraphs.

\section{C.A Essential supremum w.r.t $G_{T}^{*}$}

Definition C.1 Let $\Gamma$ be a subset of $L_{d}^{0}\left(R^{d}, \mathcal{F}_{T}\right)$. When existence holds, $\hat{\gamma}=\left(G_{T}^{*}, \mathcal{F}_{T}\right)$ - $\operatorname{esssup} \Gamma$ is the unique $\mathcal{F}_{T}$-measurable random variable satisfying the following statements

$$
\begin{aligned}
& \hat{\gamma} \geq_{G_{T}^{*}} \gamma \text { a.s., } \quad \forall \gamma \in \Gamma, \\
& \gamma \in L_{d}^{0}\left(\mathbf{R}^{d}, \mathcal{F}_{T}\right) \text { and } \gamma \geq_{G_{T}^{*}} \Gamma \Rightarrow \gamma \geq_{G_{T}^{*}} \hat{\gamma} \text { a.s. }
\end{aligned}
$$

Remark C.2 When existence of $\left(G_{T}^{*}, \mathcal{F}_{T}\right)$ - esssup $\Gamma$ holds, its uniqueness follows immediately from the fact that $G_{T}^{*}$ is proper. To alleviate the notations we shall sometimes denote esssup $\Gamma$ instead of $\left(G_{T}^{*}, \mathcal{F}_{T}\right)$ - esssup $\Gamma$ for a given set $\Gamma$.

For $\Gamma \subseteq L^{0}\left(\mathbf{R}^{d}, \mathcal{F}_{T}\right)$ denote by $|\Gamma|=\{|\gamma|, \gamma \in \Gamma\}$. As usual, esssup $|\Gamma|$ denotes the essential supremum of the real valued r.v. $|\gamma| \in \Gamma$. It is proved in [11] that:

Lemma C.3 Under the assumption that the generators of $G_{T}^{*}$ are linearly independent, if $\Gamma \subseteq L_{d}^{0}\left(\mathbf{R}^{d}, \mathcal{F}_{T}\right)$ is such that $\operatorname{esssup}|\Gamma|<\infty$ a.s., then $\left(G_{T}^{*}, \mathcal{F}_{T}\right)$ - $\operatorname{esssup} \Gamma$ exists.

Lemma C.4 Suppose that the generators of $G_{T}$ and $G_{T}^{*}$ are linearly independent. Let $\Gamma \subseteq L_{d}^{0}\left(\mathbf{R}^{d}, \mathcal{F}_{T}\right)$ be such $\left(G_{T}^{*}, \mathcal{F}_{T}\right)$ - esssup $\Gamma$ exists. Then for any generator $\xi^{i}$ and for any $\lambda \in L_{1}^{0}\left(\mathbf{R}_{+}, \mathcal{F}_{T}\right)$ :

$$
\left[\left(G_{T}^{*}, \mathcal{F}_{T}\right)-\operatorname{esssup} \Gamma\right] \cdot\left(\lambda \xi^{i}\right)=\operatorname{essup}\left\{\lambda \gamma \cdot \xi^{i}, \gamma \in \Gamma\right\}
$$

\section{C.B Weak duality}

Lemma C.5 The set $b a_{G_{T}, n}^{+}$is equal to: $b a_{G_{T}, n}^{+}=\left\{\varphi \in b a_{G_{t}, n}:\|\varphi\|=\varphi\left(\mathbf{1}_{d, n}\right)\right\}$.

Proof. 1. We start by showing the first inclusion: $b a_{G_{T}, n}^{+} \subseteq\left\{\varphi \in b a_{G_{t}, n}:\|\varphi\|=\varphi\left(\mathbf{1}_{d, n}\right)\right\}$. 
1.a. Let $\varphi \in b a_{G_{T}, n}^{+}$. Given that $\left\|1_{d, n}\right\|_{G_{T}, n} \leq 1$, we have $\|\varphi\| \geq\left|\varphi\left(\mathbf{1}_{d, n}\right)\right|$. Since $\mathbf{1}_{d, n} \geq_{G_{T}} 0, \varphi\left(\mathbf{1}_{d, n}\right) \geq 0$ and $\|\varphi\| \geq \varphi\left(\mathbf{1}_{d, n}\right)$.

1.b. To get the first inclusion, it remains to show that for all $X \in B_{G_{T}, n}$ with $\|X\|_{G_{T}, n} \leq 1$, we have $|\varphi(X)| \leq \varphi\left(1_{d, n}\right)$. If $\|X\|_{G_{T}, n} \leq 1$ then $X+\mathbf{1}_{d, n} \in G_{T}$ and $\mathbf{1}_{d, n}-X \in G_{T}$. As $\varphi$ is a positive linear form, $\varphi\left(\mathbf{1}_{d, n}+X\right)=\varphi\left(\mathbf{1}_{d, n}\right)+\varphi(X) \geq 0$ and $\varphi\left(\mathbf{1}_{d, n}-X\right)=\varphi\left(\mathbf{1}_{d, n}\right)-\varphi(X) \geq 0$, i.e. $|\varphi(X)| \leq \varphi\left(\mathbf{1}_{d, n}\right)$.

2. Reciprocally, assume that $\varphi \in b a_{G_{T}, n}$ is such that $\varphi\left(\mathbf{1}_{d, n}\right)=\|\varphi\|$. Let us show that $\varphi$ is a positive linear form.

If $X \geq_{G_{T}} 0$, then: $0 \leq_{G_{T}} X \leq_{G_{T}}\|X\|_{G_{T}, n} \mathbf{1}_{d, n}$ and

$$
-\frac{1}{2}\|X\|_{G_{T}, n} \mathbf{1}_{d, n} \leq_{G_{T}} X-\frac{1}{2}\|X\|_{G_{T}, n} \mathbf{1}_{d, n} \leq_{G_{T}} \frac{1}{2}\|X\|_{G_{T}, n} \mathbf{1}_{d, n} .
$$

Therefore, $\left\|X-\frac{1}{2}\right\| X\left\|_{G_{T}, n} \mathbf{1}_{d, n}\right\|_{G_{T}, n} \leq \frac{1}{2}\|X\|_{G_{T}, n}$, and

$$
\left|\varphi\left(X-\frac{1}{2}\|X\|_{G_{T}, n} \mathbf{1}_{d, n}\right)\right|=\left|\varphi(X)-\frac{1}{2}\|X\|_{G_{T}, n} \varphi\left(\mathbf{1}_{d, n}\right)\right| \leq\|\varphi\| \frac{1}{2}\|X\|_{G_{T}, n}
$$

Since $|\varphi|=\varphi\left(\mathbf{1}_{d, n}\right)$, it follows that

$$
\frac{1}{2}\|X\|_{G_{T}, n} \varphi\left(\mathbf{1}_{d, n}\right)-\varphi(X) \leq\left|\varphi(X)-\frac{1}{2}\|X\|_{G_{T}, n} \varphi\left(\mathbf{1}_{d, n}\right)\right| \leq \frac{1}{2}\|X\|_{G_{T}, n} \varphi\left(\mathbf{1}_{d, n}\right)
$$

and we infer that $\varphi(X) \geq 0$.

Lemma C.6 The set $L_{d}^{1, n}\left(G_{T}^{*}, \mathcal{F}_{T}\right):=\left\{Z \in L_{d}^{0}\left(G_{T}^{*}, \mathcal{F}_{T}\right): Z \cdot\left(\alpha^{i} \xi^{i}\right) \in L_{d}^{1} \forall i=1, \cdots, N\right\}$ satisfies

$$
\begin{aligned}
L_{d}^{1, n}\left(G_{T}^{*}, \mathcal{F}_{T}\right) & =\left\{Z \in L_{d}^{0}\left(G_{T}^{*}, \mathcal{F}_{T}\right): Z \cdot \mathbf{1}^{i} \in L_{1}^{1} \quad \forall i=1, \cdots N\right\} \\
& =\left\{Z \in L_{d}^{0}\left(G_{T}^{*}, \mathcal{F}_{T}\right): Z \cdot \mathbf{1}_{d, n} \in L_{1}^{1}\right\}
\end{aligned}
$$

Proof. Let $Z \in L_{d}^{1, n}\left(G_{T}^{*}, \mathcal{F}_{T}\right)$. Observe that $0 \leq_{G_{T}} \alpha_{i} \xi_{i} \leq_{G_{T}} \mathbf{1}_{d, n}$, it follows that $0 \leq Z \cdot\left(\alpha^{i} \xi^{i}\right) \leq Z \cdot 1_{d, n}$, and consequently $Z \cdot\left(\alpha_{i} \xi_{i}\right) \in L_{d}^{1}$. Reciprocally, let $Z \in G_{T}^{*}$ be such that $Z \cdot\left(\alpha^{i} \xi^{i}\right) \in L_{d}^{1} \forall i=1, \cdots, N$. It follows from the decomposition (4.4) that $Z \cdot 1_{d, n} \in L_{1}^{1}$. Then, $0 \leq_{G_{T}} \mathbf{1}^{i} \leq_{G_{T}} \mathbf{1}_{d, n}$ implies that $Z \cdot \mathbf{1}^{i} \in L_{1}^{1}$. This shows (C.9). Now, the equality (C.10) follows easily from (C.9).

Denote by $\operatorname{Vect} L_{d}^{1, n}\left(G_{T}^{*}, \mathcal{F}_{T}\right)$ the smallest vector space containing $L_{d}^{1, n}\left(G_{T}^{*}, \mathcal{F}_{T}\right)$. For $Z \in \operatorname{Vect} L_{d}^{1, n}\left(G_{T}^{*}, \mathcal{F}_{T}\right)$, we denote by $\phi_{Z} \in b a_{G_{T}, n}$ the linear form $\phi_{Z}: X \mapsto E[Z X]$, and $\|Z\|_{d, n}:=\left\|\phi_{Z}\right\|$.

Lemma C.7 The equality: $\operatorname{Vect} L_{d}^{1, n}\left(G_{T}^{*}, \mathcal{F}_{T}\right)=L_{d}^{1, n}\left(\mathbf{R}^{d}, \mathcal{F}_{T}\right)$ holds true.

Proof. The inclusion Vect $L_{d}^{1, n}\left(G_{T}^{*}, \mathcal{F}_{T}\right) \subseteq L_{d}^{1, n}\left(\mathbf{R}^{d}, \mathcal{F}_{T}\right)$ is straightforward. Now, consider $Z \in L_{d}^{1, n}\left(\mathbf{R}^{d}, \mathcal{F}_{T}\right)$, then we can write $Z=Z_{1}-Z_{2}$ where

$$
Z_{1}:=Z+\operatorname{esssup}\{-Z, 0\} \text { and } Z_{2}:=\operatorname{esssup}\{-Z, 0\} .
$$


Notice that

$$
Z_{2} \cdot \mathbf{1}_{d, n}=\sum_{i=1}^{N} Z_{2} \cdot \alpha^{i} \xi^{i}=\sum_{i=1}^{N} \operatorname{esssup}\left\{-Z \cdot \alpha^{i} \xi^{i}, 0\right\}
$$

where the last equality follows from Lemma C.4. Hence $Z_{2} \cdot \mathbf{1}_{d, n} \leq \sum_{i=1}^{N}\left|Z \cdot \alpha^{i} \xi^{i}\right|$. Using Lemma C.6, we get: $Z_{2} \in L_{d}^{1, n}\left(G_{T}^{*}, \mathcal{F}_{T}\right)$ and $Z_{2} \cdot \alpha^{i} \xi^{i} \in L_{1}^{1}$ for all $i=1, \cdots, N$. Finally, since $Z_{1}=Z+Z_{2}$, we deduce that $Z_{1}$ is also in $L_{d}^{1, n}\left(G_{T}^{*}, \mathcal{F}_{T}\right)$.

Lemma C.8 If $Z \in \operatorname{Vect} L_{d}^{1, n}\left(G_{T}^{*}, \mathcal{F}_{T}\right)$, then

$$
\|Z\|_{d, n} \leq E\left[\left(G_{T}^{*}, \mathcal{F}_{T}\right)-\operatorname{esssup}\{-Z, Z\}\right] \mathbf{1}_{d, n} \leq \sum_{i=1}^{N} E\left[\left|Z \cdot\left(\alpha^{i} \xi^{i}\right)\right|\right] .
$$

Proof. We may write $Z=Z_{1}-Z_{2}$ where $Z_{1}, Z_{2} \in L_{d}^{1}\left(G_{T}^{*}, \mathcal{F}_{T}\right)$. For $X \in B_{G_{T}, n}$ with $\|X\|_{G_{T}, n}=1$, we have $\left|\phi_{Z}(X)\right| \leq E\left[Z_{1}\right] \mathbf{1}_{d, n}+E\left[Z_{2}\right] \mathbf{1}_{d, n}=E[Z] \mathbf{1}_{d, n}+2 E\left[Z_{2}\right] \mathbf{1}_{d, n}$. Then

$$
\|Z\|_{d, n}=\left\|\phi_{Z}\right\|_{d, n} \leq E[Z] \mathbf{1}_{d, n}+2 \min _{Z_{2}} E\left[Z_{2}\right] \mathbf{1}_{d, n} .
$$

Since $Z_{2}=-Z+Z_{1}$, we have $Z_{2} \geq_{G_{T}^{*}}-Z$ and $Z_{2} \geq_{G_{T}^{*}} 0$, hence $Z_{2} \geq_{G_{T}^{*}} \operatorname{esssup}\{-Z, 0\}$. Moreover, $\mathbf{1}_{d, n} \in G_{T}$, then

$$
\begin{aligned}
E\left[Z \mathbf{1}_{d, n}\right]+2 \min _{Z_{2}} E\left[Z_{2} \mathbf{1}_{d, n}\right] & =E\left[Z \mathbf{1}_{d, n}\right]+2 E\left[\operatorname{esssup}\{-Z, 0\} \mathbf{1}_{d, n}\right] \\
& =E[Z+\operatorname{esssup}\{-2 Z, 0\}] \mathbf{1}_{d, n}=E\left[\operatorname{esssup}\{-Z, Z\} \mathbf{1}_{d, n}\right]
\end{aligned}
$$

and we conclude to the first required inequality. Then, the second inequality follows from the fact that $\mathbf{1}_{d, n}=\sum_{i=1}^{N} \alpha^{i} \xi^{i}$ and the Lemma C.4.

Proposition C.9 The normed space $\left(L_{d}^{1, n}\left(\mathbf{R}^{d}, \mathcal{F}_{T}\right),\|\cdot\|_{d, n}\right)$ is a Banach space of ba $a_{G_{T}, n}$.

Proof. Consider a Cauchy sequence $\left(Z_{k}\right)_{k \geq 1}$ in $\left(L_{d}^{1, n}\left(\mathbf{R}^{d}, \mathcal{F}_{T}\right),\|\cdot\|_{d, n}\right)$. Recall, that by definition of the norm $\|\cdot\|_{d, n}$, this means that the sequence of linear forms $\left(\phi_{Z_{k}}\right)_{k \geq 1}$ is a Cauchy sequence in $b a_{G_{T}, n}$.

step 1. We define

$$
\chi_{m, k}^{i}:=\mathbf{1}_{\left\{A_{m, k}\right\}} \mathbf{1}^{i}-\mathbf{1}_{\left\{\Omega \backslash A_{m, k}\right\}} \mathbf{1}^{i} \text { where } A_{m, n}^{i}:=\left\{\left(Z_{m}-Z_{k}\right) \cdot \mathbf{1}^{i} \geq 0\right\} .
$$

We then verify for $i=1, \cdots n$ that

$$
E\left|\left(Z_{m}-Z_{k}\right) \cdot \mathbf{1}^{i}\right|=\left|\phi_{\left(Z_{m}-Z_{k}\right)}\left(\chi_{m, k}^{i}\right)\right| \leq\left\|\phi_{Z_{m}}-\phi_{Z_{k}}\right\|_{d, n} .
$$

Therefore, for all $i=1, \cdots, n$, the sequence $\left(Z_{k} \cdot \mathbf{1}^{i}\right)_{k \geq 1}$ is a Cauchy sequence in $L_{1}^{1}$, hence converges to some $Z_{\infty}^{i} \in L_{1}^{1}$.

step 2. Now, we define

$$
Y_{m, k}^{i}:=\mathbf{1}_{\left\{B_{m, k}\right\}}\left(\alpha^{i} \xi^{i}\right)-\mathbf{1}_{\left\{\Omega \backslash B_{m, k}\right\}}\left(\alpha^{i} \xi^{i}\right) \quad \text { where } \quad B_{m, n}^{i}:=\left\{\left(Z_{m}-Z_{k}\right) \cdot\left(\alpha^{i} \xi^{i}\right) \geq 0\right\} .
$$


For this sequence $\left(Y_{m, k}^{i}\right)_{k, m}$ we verify for $i=1, \cdots N$ that

$$
E\left|\left(Z_{m}-Z_{k}\right) \cdot\left(\alpha^{i} \xi^{i}\right)\right|=\left|\phi_{\left(Z_{m}-Z_{k}\right)}\left(Y_{m, k}^{i}\right)\right| \leq\left\|\phi_{Z_{m}}-\phi_{Z_{k}}\right\|_{d, n}
$$

Therefore, for all $i=1, \cdots, N$, the sequence $\left(Z_{k} \cdot\left(\alpha^{i} \xi^{i}\right)\right)_{k \geq 1}$ is a Cauchy sequence in $L_{1}^{1}$, hence converges in $L_{1}^{1}$.

From steps 1. \& 2. we deduce that, along a subsequence the convergences hold almost surely.

step 3. Let us write $Z_{k}=Z_{k}^{1}-Z_{k}^{2}$ where $Z_{k}^{2}:=\operatorname{esssup}\left\{-Z_{k}, 0\right\} \in G_{T}^{\star}$. We have for $i=1, \cdots, N$

$$
0 \leq Z_{k}^{2} \cdot\left(\alpha^{i} \xi^{i}\right)=\operatorname{esssup}\left\{-Z_{k} \cdot\left(\alpha^{i} \xi^{i}\right), 0\right\} \leq\left|Z_{k} \cdot\left(\alpha^{i} \xi^{i}\right)\right|
$$

3.a. We state that $\liminf _{k}\left|Z_{k}^{2}\right|<\infty$ almost surely. Indeed, in the contrary case, we consider the event

$$
\tilde{\Omega}=\left\{\liminf _{k}\left|Z_{k}^{2}\right|=+\infty\right\}
$$

and assume that $\tilde{\Omega}$ is non-null. Then define the sequence: $\tilde{Z}_{k}^{2}:=\frac{Z_{k}^{2}}{1+\left|Z_{k}^{2}\right|}$. We may assume that $\left(\tilde{Z}_{k}^{2}\right)_{k}$ converges along a random subsequence to some $\tilde{Z}^{2}$ such that: $\left|\tilde{Z}^{2}\right|=1$ on $\tilde{\Omega}$. In particular $\tilde{Z}^{2} \in G_{T}^{*} \backslash\{0\}$ in $\tilde{\Omega}$. On the other hand, the inequality (C.11) implies that $\tilde{Z}^{2} \cdot\left(\alpha^{i} \xi^{i}\right)=0$ for all $i=1, \cdots, N$, hence $\tilde{Z}^{2} \cdot \mathbf{1}_{d, n}=0$ on the non-null set $\tilde{\Omega}$. This is in contradiction with $\tilde{Z}^{2} \in G_{T}^{*} \backslash\{0\} \subseteq \operatorname{int}\left(\mathbf{R}_{+}^{d}\right)$ on $\tilde{\Omega}$.

3.b. Using the fact that $Z_{k}^{1}=Z_{k}+Z_{k}^{2}$, an analogous argument allows to state that $\liminf _{k}\left|Z_{k}^{1}\right|<\infty$ almost surely.

3.c. We conclude that $\lim \inf _{k}\left|Z_{k}\right|<\infty$. Consequently, $\left(Z_{k}\right)$ converges to some $Z$ along a random subsequence and $\left(Z_{k} \cdot \alpha^{i} \xi^{i}\right)$ converges in $L_{1}^{1}$ to $\left(Z \cdot \alpha^{i} \xi^{i}\right)$ for all $i=1, \cdots, N$. Recall from Lemma C.8 that

$$
\left\|\phi_{Z_{k}}-\phi_{Z}\right\|_{d, n} \leq \sum_{i=1}^{N} E\left[\left|\left(Z_{k}-Z\right) \cdot\left(\alpha^{i} \xi^{i}\right)\right|\right] .
$$

This alows to conclude that $Z_{k}$ converges to $Z$ in $\left(L_{d}^{1, n}\left(\mathbf{R}^{d}, \mathcal{F}_{T}\right),\|\cdot\|_{d, n}\right)$.

\section{C.C The proof of Proposition 4.7}

The Krein-Smulian theorem asserts that a convex set in the dual to a Banach space is weak closed (i.e. here $\sigma\left(B_{G_{T}, n}\left(\mathbf{R}^{d}, \mathcal{F}_{T}\right), L_{1}^{1}\left(\mathbf{R}^{d}, \mathcal{F}_{T}\right)\right)-$ closed) if and only if its intersection with any ball centered at the origin is weak closed. So it is sufficient to state Proposition 4.7 for $C$ is bounded in $B_{G_{T}, n}$.

1. We start by the first implication. We assume that $C$ is weakly closed, and we consider a sequence $X^{m} \in C$ which converges to $X$ in probability. We have to show that $X \in C$. Since $C$ is bounded, there exists $c>0$ such that $\left|Z X^{m}\right| \leq c Z \mathbf{1}_{d, n}$ whatever $Z \in L_{d}^{1}\left(G_{T}^{*}, \mathcal{F}_{T}\right)$. So, by the Lebesgue theorem, for all $Z \in L_{d}^{1}\left(G_{T}^{*}, \mathcal{F}_{T}\right)$, $E\left[Z X^{m}\right]$ converges to $E[Z X]$. Hence $X^{m}$ weakly converges to $X$ and $X \in C$. 
2. Reciprocally, assume that $X^{m} \in C$ weakly converges to $X$. Since $C$ is bounded, there exists a constant $c$ independent of $m$ such that $-c \mathbf{1}_{d, n} \leq_{G_{T}} X^{m} \leq_{G_{T}} c \mathbf{1}_{d, n}$. As $G_{T}$ is proper, we deduce that $\sup _{m}\left|X^{m}\right|<\infty$ a.s. This implies, via the Von Weizsäcker Theorem 5.2.2 [10], that there exists a subsequence $X^{m_{j}}$ which is Césaro convergent to some $X^{\infty} \in C$ almost surely, that is $Y^{n}:=\frac{1}{n} \sum_{j=1}^{n} X^{m_{j}}$ converges to $X^{\infty}$ a.s. Therefore, by the same arguments as in step 1 ., $Y^{n}$ weakly converges to $X^{\infty}=X$. And we conclude that $X^{\infty}=X \in C$.

\section{References}

[1] Ph. Artzner, F.Delbaen, J-M Eber and D. Heath (1998). Coherent Measures of Risk. Finance and Stochastics 9 , 203-228.

[2] Ph. Artzner, F.Delbaen, J-M Eber, D. Heath and Hyejin Ku (2007). Coherent multiperiod risk adjusted values and Bellman's principle. Annals of Operations Research 152,1, 5-22.

[3] De Valliere D., Denis E. and Kabanov Y.(2009). Hedging of American Options under Transaction Costs . Finance and Stochastics 13 , 1, 105-119.

[4] F. Delbaen (2000). Coherent risk measures on general probability spaces. Advances in finance and stochastics: essays in honour of Dieter Sondermann.

[5] F. Feinstein and B. Rudloff (2012) Set-Valued Dynamic Risk Measure. Preprint.

[6] Hamel A., Heyde F. (2010). Duality for set-valued measures of risk, SIAM Journal of financial Mathematics, 1, 66-95.

[7] Hamel A., Heyde F., Rudlof B (2011). Set-valued risk measures for conical market models, Mathematics and financial economics, 5, 1, 1-28.

[8] R. Gianin (2006). Risk Measures via g-expectations, Insurance: Mathematics and Economics, 39, 1, 19-34.

[9] Jouini E., Meddeb M. and Touzi N. (2004) Vector valued coherent risk measures. Finance and Stochastics $8,4,531-552$.

[10] Kabanov Y., Safarian M (2009) Markets with Transaction Costs. Mathematical Theory. Springer-Verlag,

[11] Kabanov Y., Lépinette E. Essential Supremum with respect to a random partial order. Preprint available at hal .archives-ouvertes .fr/hal-00608856/.

[12] Riedel F (2004). Dynamic Coherent Risk Measures. Stochastic Processes and Their Applications. 112, 185-200.

[13] Hamel A., Heyde F., Rudlof B. (2011). Set-valued risk measures for conical market models Mathematics and financial economics, 5, 1, 1-28. 